\title{
The OTELO survey
}

\section{Demography, morphology, IR luminosity and environment of AGN hosts}

Marina Ramón-Pérez ${ }^{1,2}$, Ángel Bongiovanni ${ }^{1,2,3}$, Ana María Pérez García ${ }^{4,3}$, Jordi Cepa ${ }^{1,2,3}$, Jakub Nadolny ${ }^{1,2}$, Irene Pintos-Castro ${ }^{5}$, Maritza A. Lara-López ${ }^{6}$, Emilio J. Alfaro ${ }^{7}$, Héctor O. Castañeda ${ }^{8}$, Miguel Cerviño ${ }^{4,1,7}$, José A. de Diego 9 , Mirian Fernández-Lorenzo ${ }^{7}$, Jesús Gallego ${ }^{10}$, J. Jesús González ${ }^{9}$, J. Ignacio González-Serrano ${ }^{11,3}$, Iván Oteo Gómez ${ }^{12,13}$, Ricardo Pérez Martínez ${ }^{14,3}$, Mirjana Pović ${ }^{15,7}$, and Miguel Sánchez-Portal ${ }^{16,3}$

${ }^{1}$ Instituto de Astrofísica de Canarias (IAC), 38200 La Laguna, Tenerife, Spain e-mail: bongio@iac.es

2 Departamento de Astrofísica, Universidad de La Laguna (ULL), 38205 La Laguna, Tenerife, Spain

3 Asociación Astrofísica para la Promoción de la Investigación, Instrumentación y su Desarrollo, ASPID, 38205 La Laguna, Tenerife, Spain

${ }^{4}$ Centro de Astrobiología (CSIC/INTA), ESAC Campus, 28692 Villanueva de la Cañada, Madrid, Spain

5 Department of Astronomy \& Astrophysics, University of Toronto, Canada

${ }^{6}$ DARK, Niels Bohr Institute, University of Copenhagen, Lyngbyvej 2, Copenhagen 2100, Denmark

7 Instituto de Astrofísica de Andalucía, CSIC, 18080 Granada, Spain

8 Departamento de Física, Escuela Superior de Física y Matemáticas, Instituto Politécnico Nacional, Ciudad de México, Mexico

9 Instituto de Astronomía, Universidad Nacional Autónoma de México, Ciudad de México, Mexico

10 Departamento de Física de la Tierra y Astrofísica, Instituto de Física de Partículas y del Cosmos, IPARCOS. Universidad Complutense de Madrid, 28040 Madrid, Spain

11 Instituto de Física de Cantabria (CSIC-Universidad de Cantabria), 39005 Santander, Spain

12 Institute for Astronomy, University of Edinburgh, Royal Observatory, Blackford Hill, Edinburgh EH9 3HJ, UK

13 European Southern Observatory, Karl-Schwarzschild-Str. 2, 85748 Garching, Germany

14 ISDEFE for European Space Astronomy Centre (ESAC)/ESA, PO Box 78, 28690 Villanueva de la Cañada, Madrid, Spain

15 Ethiopian Space Science and Technology Institute (ESSTI), Entoto Observatory and Research Center (EORC), Astronomy and Astrophysics Research Division, PO Box 33679, Addis Ababa, Ethiopia

16 Instituto de Radioastronomía Milimétrica (IRAM), Av. Divina Pastora 7, Núcleo Central, 18012 Granada, Spain

Received 24 April 2018 / Accepted 25 March 2019

\begin{abstract}
Aims. We take advantage of the capabilities of the OSIRIS Tunable Emission Line Object (OTELO) survey to select and study the AGN population in the field. In particular, we aim to perform an analysis of the properties of these objects, including their demography, morphology, and IR luminosity. Focusing on the population of $\mathrm{H} \alpha$ emitters at $z \sim 0.4$, we also aim to study the environments of AGN and non-AGN galaxies at that redshift.

methods. We make use of the multiwavelength catalogue of objects in the field compiled by the OTELO survey, unique in terms of minimum flux and equivalent width. We also take advantage of the pseudo-spectra built for each source, which allow the identification of emission lines and the discrimination of different types of objects.

Results. We obtained a sample of 72 AGNs in the field of OTELO, selected with four different methods in the optical, X-rays, and mid-infrared bands. We find that using X-rays is the most efficient way to select AGNs. An analysis was performed on the AGN population of OTELO in order to characterise its members. At $z \sim 0.4$, we find that up to $26 \%$ of our $\mathrm{H} \alpha$ emitters are AGNs. At that redshift, AGNs are found in identical environments to non-AGNs, although they represent the most clustered group when compared to passive and star-forming galaxies. The majority of our AGNs at any redshift were classified as late-type galaxies, including a $16 \%$ proportion of irregulars. Another 16\% of AGNs show signs of interactions or mergers. Regarding the infrared luminosity, we are able to recover all the luminous infrared galaxies (LIRGs) in the field of OTELO up to $z \sim 1.6$. We find that the proportion of LIRGs and ultra-luminous infraed galaxies (ULIRGs) is higher among the AGN population, and that ULIRGs show a higher fraction of AGNs than LIRGs.
\end{abstract}

Key words. surveys - galaxies: active - galaxies: statistics - infrared: galaxies - X-rays: galaxies

\section{Introduction}

Galaxies hosting an active galactic nucleus (AGN) show an intense activity in a small, concentrated nuclear region, which makes them much brighter than inactive galaxies of the same Hubble type. Unlike star-forming galaxies, the intense activity of an AGN has a non-stellar origin, although both types of objects display strong emission lines in their spectra. The enormous luminosity of AGNs makes them easily recognisable at great cosmological distances, therefore their study gives us clues about the formation and evolution of galaxies in the Universe (Blandford et al. 1990). Moreover, the analysis of AGN morphologies, their environmental dependencies, and their connection to other relevant astrophysical objects such as luminous and 
ultra-luminous infrared galaxies (LIRGs and ULIRGs) are also key for our understanding of galaxy evolution.

Active galactic nuclei can be selected in a variety of ways based on their different spectral properties. One of the best ways is to perform spectroscopy in the optical or infrared (IR) range, so as to determine if the underlying ionizing continuum is of stellar type or rather follows a power law. The intensity of ultraviolet (UV) and optical emission lines can also be analysed, as proposed by Baldwin et al. (1981) and later by Veilleux \& Osterbrock (1987). This method is one of the most reliable ones, but its completeness is difficult to evaluate since it depends on the signal-to-noise ratio $(\mathrm{S} / \mathrm{N})$ of the spectra and the redshift of the emission lines (Mushotzky 2004).

When spectroscopy is not available, other selection techniques must be used. Some of these alternative methods imply looking at the galaxy mid-infrared (MIR) colours (Lacy et al. 2004; Stern et al. 2005; Donley et al. 2012). One of the main features of AGNs is the power-law continuum that generally dominates their spectrum from UV to $\sim 5 \mu \mathrm{m}$. On the contrary, star-forming galaxies exhibit a blackbody-shaped continuum due to their stellar populations in this range, with a peak around $\sim 1.6 \mu \mathrm{m}$. As a consequence, AGNs tend to be redder than normal galaxies in the MIR. By using IR colours, one can obtain information about the underlying continuum in a spectrum and detect objects whose spectral energy distribution (SED) does not decline in the red side of the stellar peak. The great advantage of MIR selection of AGNs is that it permits to detect even those objects obscured by interstellar gas or by dust that cannot be seen in X-rays or in the optical. However, when compared to other bands, images in IR may sometimes suffer from poorer spatial resolution. Another drawback is that at intermediate luminosities, AGN selection in the IR seems to be biased towards unobscured AGNs (Messias et al. 2014).

Other AGN-selection techniques focus on the X-ray emission, as it is a very good indicator of nuclear activity in galaxies (Mushotzky 2004). In fact, AGNs are believed to be the prevailing astronomical objects contributing to the cosmic X-ray background (Della Ceca et al. 2004). In the surveys carried out with the Chandra and XMM-Newton spatial observatories for instance, the majority of the extragalactic X-ray sources that were found were AGNs (Brandt et al. 2004). The strong X-ray emission of those objects is produced in the central regions of the accretion disc surrounding the black hole.

Due to the diversity of AGN types, a specific technique may correctly select an AGN population while missing others. For instance, a selection based on X-ray or optical emission can miss the population of obscured (either by interstellar gas or by dust) AGNs, unlike an IR-photometry-based method. On the other hand, X-ray emission is a powerful tool to select low-luminosity AGNs or AGNs hiding behind larger hydrogen column densities than those found by optical methods. That is why a multiwavelength approach is preferable in order to obtain reliable unbiased AGN datasets.

In recent years, the use of tunable filters (TFs) in large telescopes has begun to stand out as an efficient way of obtaining low-resolution spectroscopy of a large number of sources simultaneously, and also exploring the sky at deeper magnitudes (see Bongiovanni et al. 2019, and references therein). This technique is particularly useful for the detection of emitting objects even at high redshifts. The OSIRIS Tunable Emission Line Object project, OTELO ${ }^{1}$, is an ambitious emission-line survey which makes use of the red TF of the OSIRIS instrument (Cepa et al.

1 http://www.iac.es/proyecto/otelo
2003), installed in the $10.4 \mathrm{~m}$ Gran Telescopio Canarias (GTC), currently the largest fully steerable optical reflecting telescope in the world (Álvarez et al. 1998). OTELO is a blind tomography that samples the spectral range (9070-9280 $\AA$ ) every $6 \AA$ with a full-width at half maximum (FWHM) of about $12 \AA$, allowing for the observation of emission lines in well-defined redshift windows in a selected area of $7.5^{\prime} \times 7.4^{\prime}$ in the Extended Groth Strip. In particular, the $\mathrm{H} \alpha+[\mathrm{NII}]$ lines are observed at $z \sim 0.4$.

OTELO is the deepest emission-line survey to date, with unique detection limits in terms of minimum flux and equivalent width (EW; Ramón-Pérez et al. 2019). Moreover, a large multiwavelength catalogue of all the sources detected in the field, with data ranging from X-rays to far-infrared (FIR), has been compiled in Bongiovanni et al. (2019). This catalogue contains 11237 entries and is $50 \%$ complete at AB magnitude 26.38. A summary of the available bands in this catalogue is shown in Table 1.

The multiwavelength catalogue of OTELO is a fundamental tool for the identification of all the AGNs in the field using different selection methods in different wavelength bands. Moreover, the pseudo-spectra (PS) directly derived for each source in the field (see Bongiovanni et al. 2019 for a description) allow us to go one step further in the identification of AGNs through their emission-lines. It also permits the identification of all the $\mathrm{H} \alpha$ emitters in the field (both AGNs and non-AGNs).

In this work, we therefore aim to take advantage of the capabilities of OTELO to study the AGN population in the field. In particular, we aim to perform an analysis of the properties of these objects, including their demography and morphology. Focusing on the population of $\mathrm{H} \alpha$ emitters at $z \sim 0.4$, an attempt will be made to study the environments of AGNs and non-AGNs at that redshift.

This paper is organised as follows. In Sect. 2 the selection of AGNs in the field by different methods is explained. The analysis of AGNs, including their environment, morphology, and the identification of LIRGs and ULIRGs is described in Sect. 3. Finally, Sect. 4 summarises the main conclusions of this work.

In this paper we assume a standard $\Lambda$-cold dark matter cosmology with $\Omega_{\Lambda}=0.69, \Omega_{\mathrm{m}}=0.31$, and $H_{0}=67.8 \mathrm{~km} \mathrm{~s}^{-1} \mathrm{Mpc}^{-1}$, as extracted from Planck Collaboration XX (2016).

\section{Selection of AGNs}

We took advantage of the multiwavelength data and PS available for OTELO sources. A summary of the photometric bands included in the catalogue of OTELO can be found in Table 1. Three different techniques were used in order to select AGNs. The first one targets the AGN optical emission and uses a diagnostic diagram to separate them from star-forming galaxies (SFGs). The second one employs the X-ray-to-optical-flux ratio $(\mathrm{X} / \mathrm{O})$. Finally, the third one uses MIR colour-colour diagrams.

\section{1. $A G N$ at $z \sim 0.4$}

The first method to select AGNs benefits from the potential of the OTELO survey to identify emitting objects. The flux excess measured on the PS built for every object in the catalogue of OTELO, or its location in the appropriate colourmagnitude diagram, together with the photometric redshift estimation, allow us to make a preliminary identification of the emission-line source candidates per volume of universe explored (see Bongiovanni et al. 2019). In this case, a range of $0.3 \leq$ photo- $z \leq 0.5$ was chosen in order to ensure a sample of potential 
Table 1. Available bands in OTELO's multiwavelength catalogue and their corresponding original catalogues (see Bongiovanni et al. 2019 for more details).

\begin{tabular}{lccc}
\hline \hline & Catalogue & Bands & Reference \\
\hline X-rays & Chandra & $0.5-7 \mathrm{keV}$ & Pović et al. (2009) \\
Ultraviolet & GALEX & $N U V, F U V$ & Morrissey et al. (2007) \\
Mid-infrared & Spitzer/IRAC & $3.6,4.5,5.8, \& 8 \mu \mathrm{m}$ & Barro et al. (2011) \\
Far-infrared (I) & Spitzer/MIPS \& Herschel/PACS & $24,100 \& 160 \mu \mathrm{m}$ & Lutz et al. (2011) \\
Far-infrared (II) & Herschel/SPIRE & $250,300 \& 500 \mu \mathrm{m}$ & Roseboom et al. (2010) \\
\hline
\end{tabular}

$\mathrm{H} \alpha$ candidates that is as complete as possible. This guess redshift range also takes into account a reported photo- $z$ accuracy $|\Delta z| /(1+z) \leq 0.2$. Subsequently, these candidates were individually examined using a collaborative web-based data visualisation facility that includes a line identifier tool in order to assign scaled likelihood values to the possible line identities, and therefore reliable redshifts. The probability of a given candidate to belong (or not) to the OTELO $\mathrm{H} \alpha$ window was then calculated by comparing and weighting the different values of redshift and the corresponding likelihoods assigned to this object.

Following this methodology, we obtained a sample of 46 sources whose PSs show an emission compatible with the $\mathrm{H} \alpha+[\mathrm{NII}] \lambda 6584$ feature. In our case, the wavelength sampling of the PS would also enable the deblending of the $\mathrm{H} \alpha$ and [NII] lines and the measurement of fluxes and EWs (Lara-López et al. 2011). This makes it possible to use optical diagnostics aimed to discriminate between AGNs and starburst galaxies. A further description of the selection of $\mathrm{H} \alpha$ emitters at $z \sim 0.4$ in the field of OTELO is given in Ramón-Pérez et al. (2019).

\subsubsection{Broad-line AGNs}

First of all, we selected broad-line AGNs (BLAGNs) from our sample of $46 \mathrm{H} \alpha$ emitters. Broad-line AGNs show permitted lines with widths of thousands of kilometers per second. In comparison, narrow-line AGNs (NLAGNs) have line widths of only a few hundred kilometers per second or less. Their selection is described in Sect. 2.1.2.

In order to check the aspect of broad-lines when observed through OSIRIS tunable filters, we first performed a simulation using two real spectra of BLAGNs (Seyferts 1.5 NGC 3516, see Arribas et al. 1997, and NGC 4151, see Kaspi et al. 1996), following the same methodology as Sánchez-Portal et al. (2015). We saw that even if the $\mathrm{H} \alpha+[\mathrm{NII}]$ emission was well reproduced in both examples, in the case of NGC 3516 the line is so broad that it becomes diluted after the convolution and the object would fail the automatic test for the detection of emission lines described in Sánchez-Portal et al. (2015). According to the results of our detection-limit simulations for the case of the $\mathrm{H} \alpha$ emission line, an approximate upper detection limit is $\sim 60 \AA$ for the FWHM of the input Gaussian ( $\mathrm{H} \alpha)$, corresponding to $\sim 84 \AA$ at $z=0.398$ and to a width of $\sim 2700 \mathrm{~km} \mathrm{~s}^{-1}$ at that redshift (Ramón-Pérez et al. 2019). The situation improves when the lines are not centred in the wavelength window of OTELO. In those cases, when the line appears close to the limiting edges of the spectral window, the pseudo-continuum is more realistically reproduced by the algorithm, favouring the detection.

BLAGN were selected on the basis of two different but equivalent criteria. The first one consisted in fitting a Gaussian to the pseudo-spectrum and determining the FWHM of the fit (as in Fig. 1). After visual inspection to discard incorrect or unclear fits, we selected as BLAGN those objects having a FWHM greater than $\sim 30 \AA$ (corresponding to $\sim 1000 \mathrm{~km} \mathrm{~s}^{-1}$ at $z \sim 0.4)$. The second criterion was used whenever the pseudospectrum could not be fitted. In those cases, we calculated the number of PS points around the maximum that exceeded half its value. Two different maxima were considered: (i) the real PS maximum and (ii) the closest PS point to the $\mathrm{H} \alpha$ line maximum, given the redshift. If at least five points around one of this maximum had a value higher than half maximum, the object was considered a BLAGN. Taking into account that the sampling interval of OTELO is $6 \AA$, both this criterion and the previous one are equivalent. In total, six $\mathrm{H} \alpha$ emitters were selected as BLAGN by one or both of these criteria. They are shown in Fig. A.1. One of these objects (the last one in the figure) showed a truncated line which prevented the fitting and the analysis. However, it was included in the final sample of BLAGN because its width is comparable to the rest of the objects selected as BLAGN, if we assume a symmetrical line.

\subsubsection{Narrow-line AGNs: measurement of equivalent width and flux of the $\mathrm{H} \alpha$ and [NII] 16584 emissions}

The first step in the process of selecting NLAGN was to subtract the continuum of the pseudo-spectra, previously calculated by a linear fit to the data points outside the emission line and visually verified. In some cases, when the continuum fit was not good enough and included part of the emission line, the continuum level was subtracted manually. The $\mathrm{H} \alpha$ and [NII] fluxes $[f(\mathrm{H} \alpha)$ and $f([\mathrm{NII}])$, respectively] were derived following the procedure described in Sánchez-Portal et al. (2015), that assumes infinitely thin lines. For each object, the redshifted position of both lines in wavelength is known. The fluxes measured in the closest scan slices to these positions ( $f_{\mathrm{H} \alpha}$ and $f_{[\mathrm{NII}]}$, respectively), correspond to a combination of both line fluxes, such as

$f_{\mathrm{H} \alpha}=T_{\mathrm{H} \alpha}(\mathrm{H} \alpha) f(\mathrm{H} \alpha)+T_{\mathrm{H} \alpha}([\mathrm{NII}]) f([\mathrm{NII}])$,

$f_{[\mathrm{NII}]}=T_{[\mathrm{NII}]}(\mathrm{H} \alpha) f(\mathrm{H} \alpha)+T_{[\mathrm{NII}]}([\mathrm{NII}]) f([\mathrm{NII}])$.

In the previous equations, T"slice" ("line") represents the TF transmission of a given slice at a given wavelength. The real $\mathrm{H} \alpha$ and [NII] fluxes can then be derived from the previous equations as follows.

$f(\mathrm{H} \alpha)=\frac{f_{\mathrm{H} \alpha} T_{[\mathrm{NII}]}([\mathrm{NII}])-f_{[\mathrm{NII}]} T_{\mathrm{H} \alpha}([\mathrm{NII}])}{T_{\mathrm{H} \alpha}(\mathrm{H} \alpha) T_{[\mathrm{NII}]}([\mathrm{NII}])-T_{\mathrm{H} \alpha}([\mathrm{NII}]) T_{[\mathrm{NII}]}(\mathrm{H} \alpha)}$,

with a similar equation for $f([\mathrm{NII}])$. Equivalent widths were then converted to rest frame using the redshift information. The distribution of $\mathrm{H} \alpha$ fluxes can be seen in Fig. 9. The median error was $\sim 12 \%$, in agreement with the simulations performed by Lara-López et al. (2011), who obtained errors below $20 \%$ for a FHWM of the OSIRIS TF of $12 \AA$ and a sampling of $6 \AA$. 

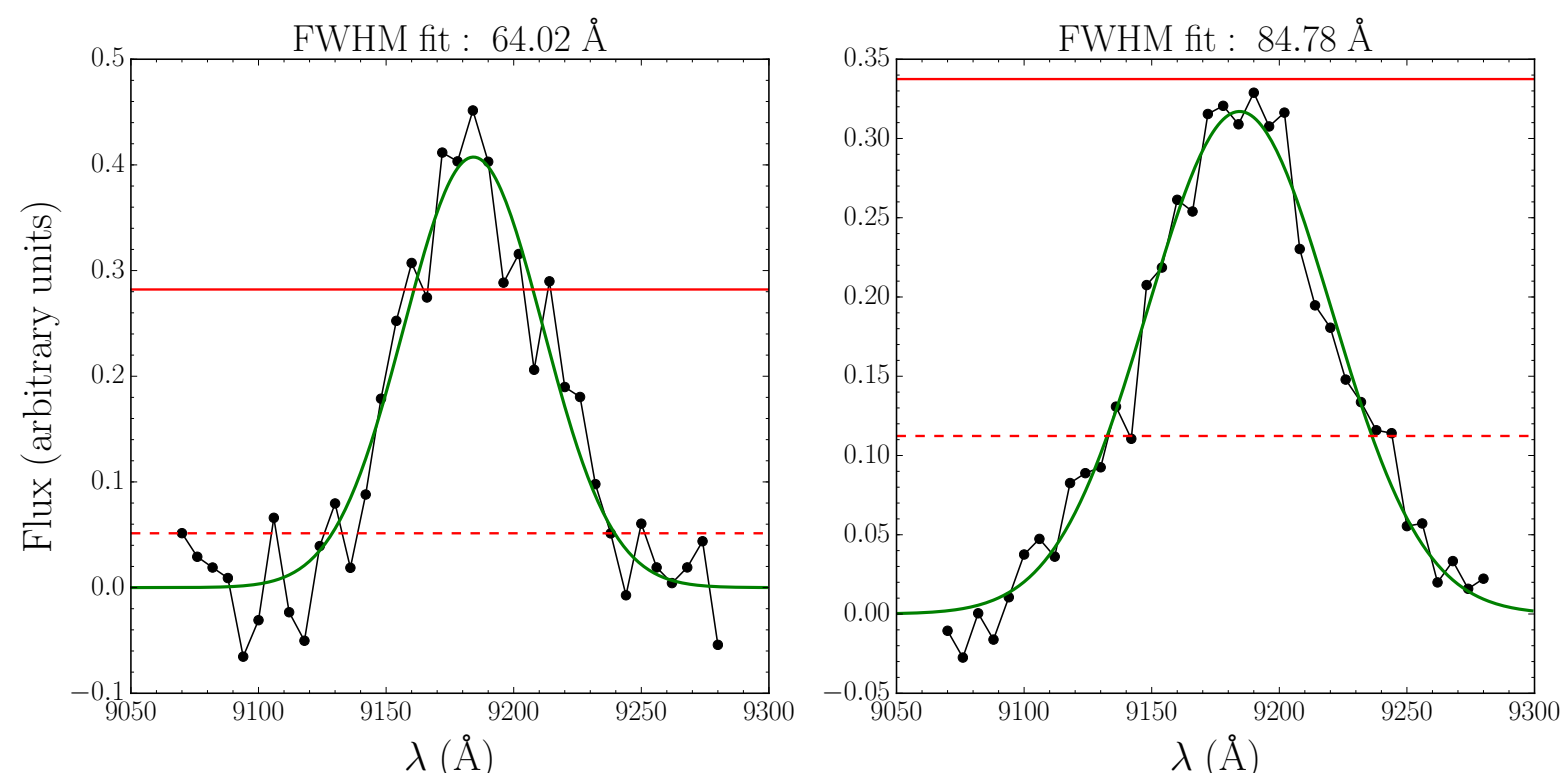

Fig. 1. Simulated pseudo-spectra (black dots) of $\mathrm{H} \alpha+[\mathrm{NII}]$ lines with different widths after being convolved to the TF spectral response. The green lines represent the best Gaussian fit to each pseudo-spectrum and the titles describe the corresponding FWHM. The red dashed lines represent the pseudo-continuum, $f_{\text {cont }}$, defined as the median value of the pseudo-spectrum points that remain within $2 \sigma$ of the median value of the whole pseudo-spectrum. The red continuous lines represent $f_{\text {cont }}+2 \sigma_{\text {cont }}$, where $\sigma_{\text {cont }}$ is the standard deviation of the pseudo-continuum points. The automatic algorithm used in Ramón-Pérez et al. (2019) efficiently detects broad lines as emitting lines for widths up to $\sim 84 \AA$. In the left panel, with a FHWM of $64.02 \AA$, the broad line is detected. On the contrary, the line in the right panel is so broad (FWHM of $84.78 \AA$ ) that it is not recognised.

However, our errors in the measurement of the [NII] line were much higher, with $60 \%$ of the objects having errors above $50 \%$. This was to be expected since the [NII] line is usually fainter than the $\mathrm{H} \alpha$ one, especially in a sample mainly composed of low-luminosity sources, as demonstrated in Ramón-Pérez et al. (2019). As noted in the following section, SFGs constitute about two thirds of the 28 (from 46) H $\alpha$ sources with [NII] fluxes effectively measured after the BLAGN segregation.

\subsubsection{Discrimination between star-forming galaxies and AGNs}

One of the most used diagnostic diagrams to discriminate between SFG and AGN hosts is the Baldwin, Phillips \& Terlevich (BPT) diagram (Baldwin et al. 1981), which uses the ratios of $[\mathrm{OIII}] / \mathrm{H} \beta$ and $[\mathrm{NII}] / \mathrm{H} \alpha$ emission lines. Other flux ratios, such as $[\mathrm{SII}](\lambda 6716+\lambda 6731) / \mathrm{H} \alpha$ or $[\mathrm{OI}] \lambda 6300 / \mathrm{H} \alpha$ are also useful for this purpose (Baldwin et al. 1981; Veilleux \& Osterbrock 1987). Unfortunately, those lines are not always available, and their fluxes cannot always be measured. Simpler diagnostic diagrams are thus needed to separate distinct classes of objects. An alternative is to use the named $\mathrm{EW} \alpha \mathrm{n} 2$ diagram, in which the $[\mathrm{OIII}] / \mathrm{H} \beta$ ratio of the BPT diagram is replaced with the $\mathrm{EW}$ of $\mathrm{H} \alpha$ at restframe (Cid Fernandes et al. 2010).

In the EW $\alpha \mathrm{n} 2$ diagram, star-forming and active galaxies occupy separate regions along the horizontal axis, while Seyferts and LINERs are differently distributed along the vertical axis. Several criteria can be used in order to select AGNs. Stasińska et al. (2006), for instance, defines pure SFGs as those objects lying in the $\log [\mathrm{NII}] / \mathrm{H} \alpha \leq-0.4$ region and AGNs as those with $\log [\mathrm{NII}] / \mathrm{H} \alpha>-0.2$. In the intermediate region, hybrid objects having both star-formation and nuclear activity are located. A similar classification for AGNs is proposed by Ho et al. (1997), while Kewley et al. (2001) are slightly more restrictive and consider pure AGNs to be those objects with log $[\mathrm{NII}] / \mathrm{H} \alpha>-0.1$. Moreover, a separation between LINERs and Seyferts can be traced at $\mathrm{EW}(\mathrm{H} \alpha)=6 \AA$ (rest-frame), according to Kewley et al. (2006).

Figure 2 shows the EW $\alpha \mathrm{n} 2$ diagram for our sample of $\mathrm{H} \alpha$ emitters. The different criteria previously described are shown. We have also traced our minimum detected $\mathrm{EW}(\mathrm{H} \alpha)$ with a probability threshold of $p \geq 0.95$ and $p \geq 0.50$, according to the results of the simulations described in Ramón-Pérez et al. (2019). In order to ensure the selection, objects with EWs below the $p \geq 0.95$ limit, including two possible LINERs, were discarded from the analysis. Accordingly, all the selected AGNs are presumably Seyfert galaxies with $\operatorname{EW}(\mathrm{H} \alpha)>0.6$. We selected all galaxies showing evidence of nuclear activity, either in composite (i.e. $\mathrm{SF}+\mathrm{AGN}$ ) galaxies or in pure active ones, following the criterion of Stasińska et al. (2006). In this way, six H $\alpha$ emitters were selected as NLAGNs. However, due to the large uncertainties in the [NII] line flux measurements, the [NII]/H $\alpha$ ratios also have large errors (the median relative error in the sample was $32 \%$, see error bars in Fig. 2) and therefore sources close to one side of the SF/AGN statistical frontier could belong to the another object type.

We search for additional insights to reinforce the results of the EW $\alpha \mathrm{n} 2$ diagnostics. Only one of the $\mathrm{H} \alpha$ emitters is included in the X-ray emitter subset (see Sect. 3.1). On the other hand, we have not found subtantial differences in optical/IR luminosity or MIR flux ratios between these subsamples of $\mathrm{H} \alpha$ emitters with an effectively measured [NII] line flux, as used in the following sections for the whole AGN population in OTELO presented here.

According to Cid Fernandes et al. (2011), the BPT-based criterion of Stasińska et al. (2006) is more of a "pure-SF" demarcation line than a line used to divide SFGs from AGNs. In this sense, and taking into account the significance of the median 


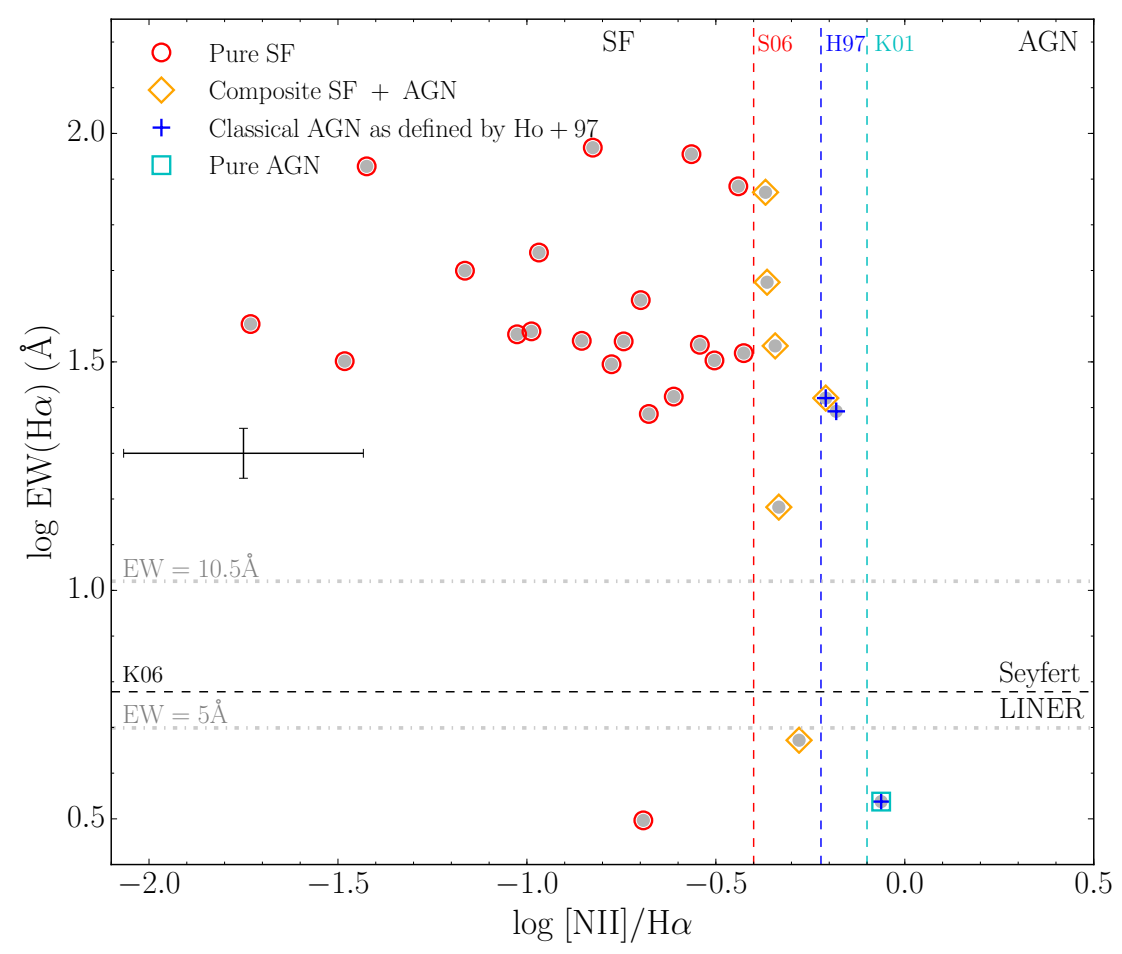

Fig. 2. EW $\alpha \mathrm{n} 2$ diagnostic diagram defined by Cid Fernandes et al. (2010) in order to distinguish SFGs from AGNs using emission lines in the optical. Pure SFGs (red circles) are separated from composite objects ( $\mathrm{SF}+\mathrm{AGN}$, orange diamonds) according to Stasińska et al. (2006) (red dashed vertical line). Classical AGNs as defined by Ho et al. (1997) (blue dashed vertical line) are displayed with blue crosses. Pure AGNs according to Kewley et al. (2001) (cyan dashed vertical line) are represented in cyan squares. The black dashed horizontal line corresponds to the Seyfert/LINER separation criterion by Kewley et al. (2006). The two grey dashed-dotted horizontal lines mark our minimum detected $\mathrm{EW}(\mathrm{H} \alpha)$ with a probability threshold of $p \geq 0.95$ for objects with a PS continuum up to $\sim 10^{-18} \mathrm{erg} \mathrm{s}^{-1} \mathrm{~cm}^{-2} \AA^{-1}$, and a $\operatorname{EW}(\mathrm{H} \alpha)$ with $p \geq 0.50$ for objects with a PS continuum up to $\sim 10^{-19} \mathrm{erg} \mathrm{s}^{-1} \mathrm{~cm}^{-2} \AA^{-1}$ (see Ramón-Pérez et al. 2019). The error bars on the left-hand side of the plot represent the median of the relative errors of our $\mathrm{H} \alpha$ sample in this space. error in the $[\mathrm{NII}] / \mathrm{H} \alpha$ ratio with respect to this boundary, more than half of the $\mathrm{H} \alpha$ with [NII] line flux measured are bona fide SFGs, and the selection of NLAGNs with this procedure should be taken with caution.

\subsection{X-ray selection}

The strong X-ray emission, produced in the central regions of the accretion disc surrounding the black hole, is a good indicator of nuclear activity in galaxies. In particular, Maccacaro et al. (1988) showed the power of the X-ray-to-optical flux ratio (X/O) to distinguish AGNs from other X-ray-emitting sources. The OTELO catalogue has information in the soft $0.5-2 \mathrm{keV}$ band from Pović et al. (2009) and Laird et al. (2009), and therefore we adopt the Szokoly et al. (2004) X/O definition:

$\mathrm{X} / \mathrm{O} \equiv \log _{10}\left(f_{\mathrm{X}} / f_{\mathrm{O}}\right) \equiv \log _{10}\left(f_{\mathrm{X}}\right)+0.4 R+5.71$,

where $f_{\mathrm{X}}$ is the $\mathrm{X}$-ray flux in the $0.5-2 \mathrm{keV}$ band $\left(\mathrm{erg} \mathrm{s}^{-1} \mathrm{~cm}^{-2}\right)$ and $R$ is the optical magnitude in Vega magnitudes.

According to Stocke et al. (1991), AGNs are typically located in the $-1<\mathrm{X} / \mathrm{O}<1$ range. At very high values of $\mathrm{X} / \mathrm{O}$ we can find not only AGN types 1 and 2 , but also clusters of galaxies at high redshift, extreme BL Lac objects, and cooling-flow galaxies. On the other hand, lower values of this ratio $(\mathrm{X} / \mathrm{O}<-1)$ in extragalactic sources include normal and star-forming galaxies, as well as low-luminosity AGNs (see Alexander et al. 2001, and references therein), some of which would account for possible composite objects. For our purpose, objects with nuclear activity and those with star formation can be separated with the $\mathrm{X} / \mathrm{O}=-1$ limit.

In Fig. 3 we plot the $\mathrm{X} / \mathrm{O}$ ratio as a function of the optical magnitude, for the sources in the OTELO catalogue that show an X-ray emission and have information in the soft band (53 out of 56). We marked 42 sources with $\mathrm{X} / \mathrm{O}>-1$ as AGNs, but regarding the typical uncertainty in the calculation of this ratio, this number could vary by $5-7 \%$ above or below this hard boundary, respectively, if the sources around it are different from

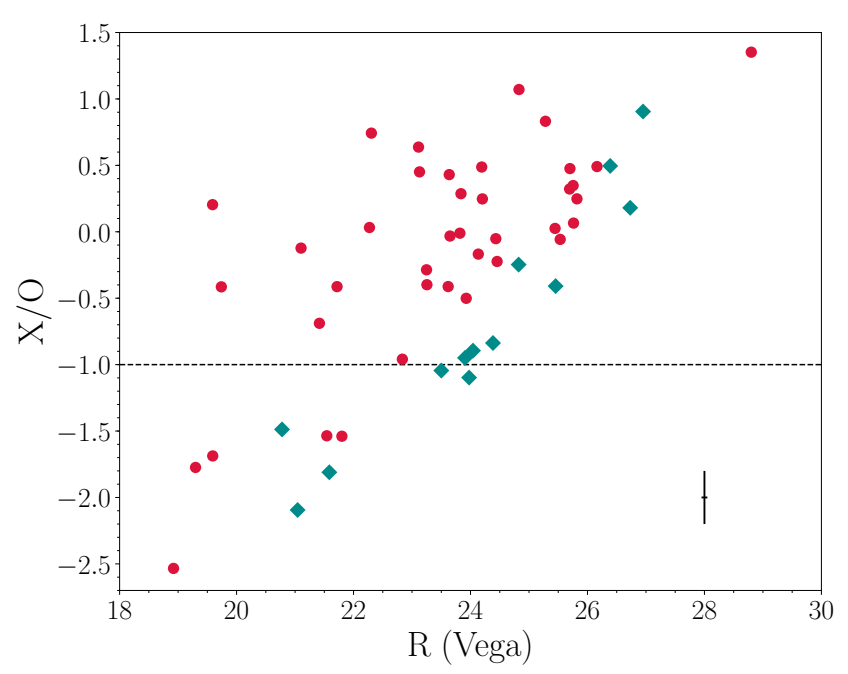

Fig. 3. X-ray-to-optical flux ratio (X/O) defined by Eq. (3), as a function of the optical band ( $R$ in Vega magnitudes) of the X-ray emitting sources in the OTELO catalogue. Red dots correspond to the sources from the original catalogue of Pović et al. (2009) with information in the soft band $(0.5-2 \mathrm{keV})$, while the green diamonds are the ones from the catalogue of Laird et al. (2009). The adopted criterion to select AGNs is $\mathrm{X} / \mathrm{O}>-1$ (dashed line). The bar represents the typical error propagated to the $\mathrm{X} / \mathrm{O}$ ratio at this boundary for this sample. A total of $42 \mathrm{AGN}$ counterparts was found regardless of the effects of this uncertainty estimation (see the text).

low-luminosity AGNs (see, for instance, Hornschemeier et al. 2001) or composite objects.

\subsection{Mid-infrared selection}

As mentioned in Sect. 1, the SED of AGNs from UV to $\sim 5 \mu \mathrm{m}$ is dominated by a power-law continuum, while SFGs tend to show a black-body spectrum that peaks at $\sim 1.6 \mu \mathrm{m}$ as a signature of 


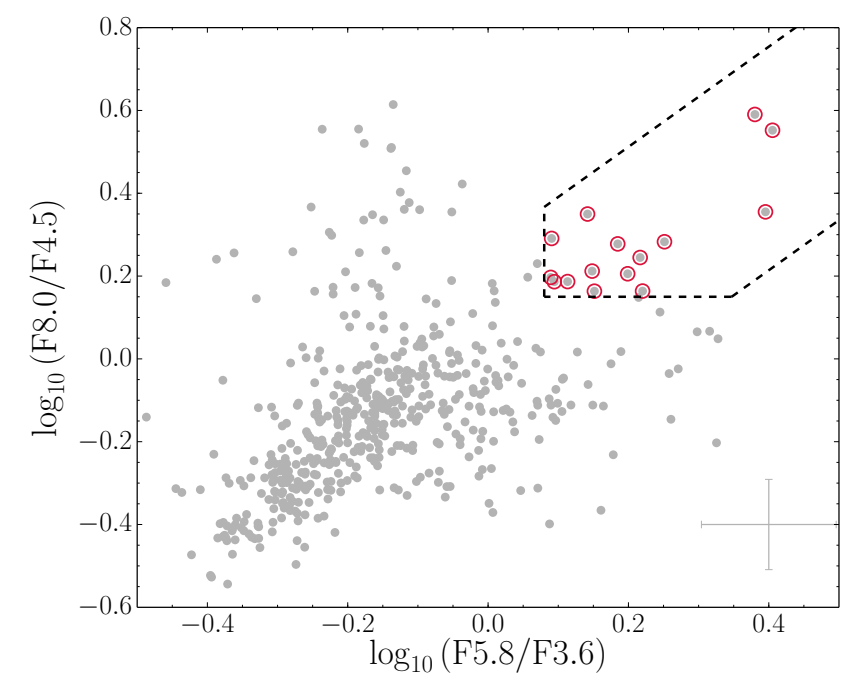

Fig. 4. Revised Spitzer/IRAC criteria from Donley et al. (2012) to separate AGNs from SFGs. The $x$ axis shows the ratio between the flux in the $5.8 \mu \mathrm{m}$ band and the flux in the $3.6 \mu \mathrm{m}$ band, while the $y$ axis depicts the ratio between the flux in the $8.0 \mu \mathrm{m}$ band and the flux in the $4.5 \mu \mathrm{m}$ band. Grey dots are all the sources in the OTELO catalogue with information in the four IRAC bands. Black dashed lines correspond to the limits set by Donley et al. (2012) to select AGNs. Red circled sources are the 15 sources selected as AGNs in this way.

the underlying stellar populations. Thus, AGNs are redder than normal galaxies in the MIR, and IR colours can help to distinguish between different galaxy spectral types.

Diagnostic diagrams to discriminate AGNs from SFGs using IR colours are very common. One of the most remarkable is the empirical criterion proposed by Stern et al. 2005. Nevertheless, the authors claim that this method may omit AGNs at redshifts between $z \sim 0.8$ and 2 and that the selection is contaminated by SFGs at high redshift. It is therefore not convenient in the case of OTELO, a survey that is not limited by redshift. Consequently, we decided to use the method of Donley et al. (2012) to select AGNs based on their MIR colours. This method makes use of the fluxes in the four Spitzer/IRAC bands (3.6, 4.5, 5.8 and $8.0 \mu \mathrm{m})$ and defines an empirical region where AGNs are found:

$x \geq 0.08$

$y \geq 0.15$

$y \geq 1.21 \times x-0.27$

$y \leq 1.21 \times x+0.27$,

where $x=\log _{10}\left(f_{5.8 \mu \mathrm{m}} / f_{3.6 \mu \mathrm{m}}\right)$ and $y=\log _{10}\left(f_{8.0 \mu \mathrm{m}} / f_{4.5 \mu \mathrm{m}}\right)$. As can be seen in Fig. 4, 15 AGNs were found in this way.

In addition, we use an alternative second MIR criterion to select AGNs in the field (the "KIM" criterion), based on the work by Messias et al. (2012). This criterion not only takes the IRAC bands into account, but also the $K_{\mathrm{s}}$ and the $24 \mu \mathrm{m}$ bands, which are also contained in the OTELO multiwavelength catalogue. The KIM criterion defines the following region where AGNs are found ("IM" criterion):

$$
\begin{aligned}
& {[8.0]-[24]>-2.9 \times([4.5]-[8.0])+2.8} \\
& {[8.0]-[24]>0.5,}
\end{aligned}
$$

where [4.5], [8.0], and [24] represent AB magnitudes in the 4.5 and $8.0 \mu \mathrm{m}$ IRAC bands and in the $24 \mu \mathrm{m}$ Spitzer/MIPS band, respectively. In addition to that, sources have to fulfill a third

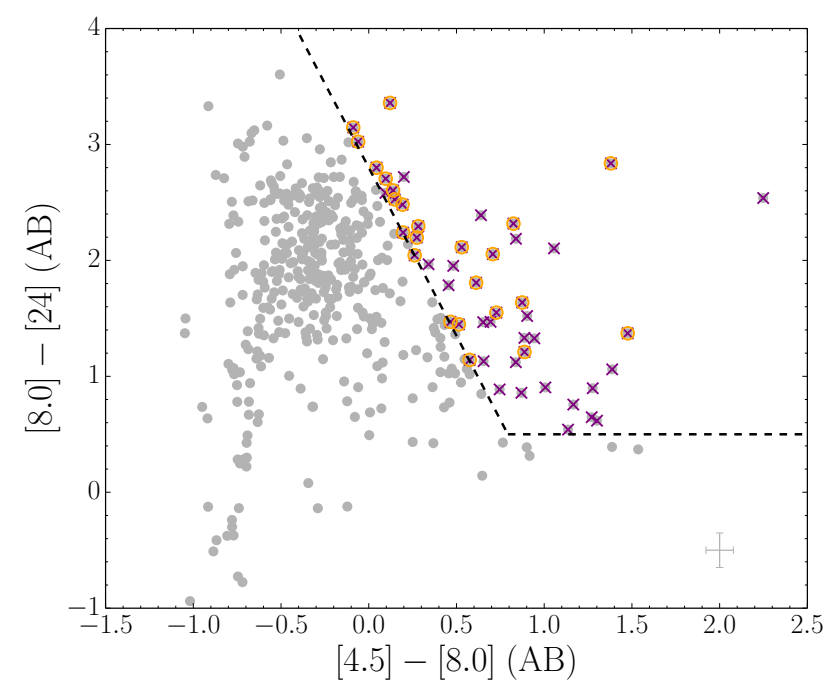

Fig. 5. IRAC+MIPS (IM) IR criteria by Messias et al. (2012) to select AGNs. Grey dots are all the OTELO sources with information in the four IRAC bands as well as in the $K_{\mathrm{s}}$ and [24] $\mu \mathrm{m}$ bands. Black dashed lines are the limits of the IM criterion (see Eq. (5)). Purple crosses represent the objects fulfilling the IM criterion. Orange circled sources represent the sources satisfying, in addition, that $K_{\mathrm{s}}-[4.5]>0$, i.e., the KIM $\left(K_{\mathrm{s}}+\mathrm{IRAC}+\mathrm{MIPS}\right)$ criterion. These are the objects selected as AGNs by the KIM criterion (24 sources).

Table 2. Summary of AGN selection.

\begin{tabular}{cccccc}
\hline \hline $\begin{array}{c}\text { Number of } \\
\text { objects }\end{array}$ & X-rays & MIR & $\begin{array}{c}\text { NLAGNs } \\
(z=0.4)\end{array}$ & $\begin{array}{c}\text { BLAGNs } \\
(z=0.4)\end{array}$ \\
\hline & 31 & $\checkmark$ & $x$ & $x$ & $x$ \\
& 11 & $\checkmark$ & $\checkmark$ & $x$ & $x$ \\
& 18 & $x$ & $\checkmark$ & $x$ & $x$ \\
& 6 & $x$ & $x$ & $\checkmark$ & $x$ \\
Total: & $\mathbf{7 2}$ & $x$ & $x$ & $x$ & $\checkmark$ \\
\hline
\end{tabular}

Notes. The first column indicates the number of objects in each group. The following columns specify the selection methods: X-rays, MIR, NLAGNs at $z=0.4$ or BLAGNs at $z=0.4$. The green checkmark means that an object at any redshift has been selected as an AGN by the corresponding method, while the red cross indicates that none of the objects have been selected by that method. Each row shows a subgroup of AGNs detected by one or more methods. The last row, in bold, indicates the total number of AGNs in each group.

condition: $K_{\mathrm{s}}-[4.5]>0$ ("K" criterion). In this way, this method minimises contamination at low redshifts from normal galaxies while effectively separating AGNs from SFGs at high redshifts, and thus can be used at all ranges of $z$. In Fig. 5, we plot the [8.0] - [24] versus [4.5] - [8.0] colours, and have selected the sources fulfilling both the IM criterion and $K_{\mathrm{S}}-[4.5]>0$. Given that OTELO has no constraints on redshift, we used the KIM criterion and selected the latter (24 sources) as AGNs. From those, ten were selected by Donley et al. (2012) criteria and 14 were new. In total, 29 AGNs were selected using IR-based methods.

Finally, Table 2 summarises the different criteria used for the selection of AGNs, and the number of objects selected in each case. In total, 72 objects were classified as AGNs. 


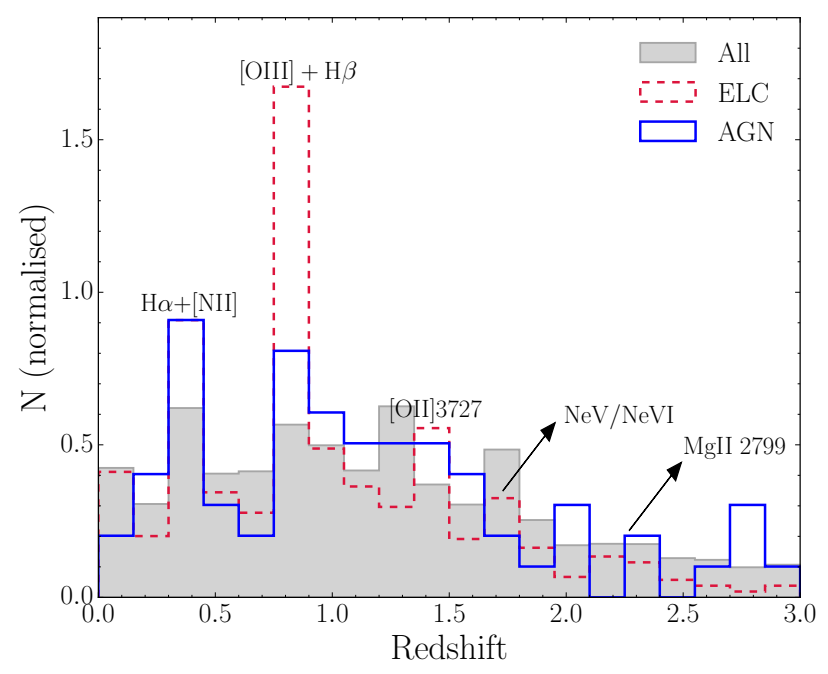

Fig. 6. Distribution of redshifts (obtained with LePhare as described in Bongiovanni et al. 2019) for the whole sample of OTELO (grey), the emitting-line candidates (ELC, shown in red; see text for details), and the AGN sample (shown in blue). Some of the most intense emission lines in this spectral interval are displayed.

\section{Analysis of AGNs}

Once the AGN population is selected from the OTELO survey, a first analysis is performed in order to gather information about its general characteristics, such as its demography and morphology. Also, the fraction of LIRGs and ULIRGs is studied. Finally, an inceptive analysis is performed for the subpopulation of AGNs at $z \sim 0.4$, which includes a study of the source environment.

\subsection{Demography}

The AGN population found in OTELO with the methods described in the previous section comprises 72 objects and represents a very small fraction of the total number of objects in the catalogue (less than 1\%). Their distribution in redshift, as obtained with LePhare (see Bongiovanni et al. 2019), can be seen in Fig. 6, together with that of the total and selected emission-line candidate (ELC) populations, which were selected using the methodology described in Sect. 2.1. It can be seen that there are more ELCs and AGNs at the redshifts corresponding to the more intense optical emission lines, which is a characteristic bias of emission-line surveys. In particular, the AGN population exhibits a peak at $z \sim 0.4$, as this is the redshift at which the $\mathrm{H} \alpha$ line appears in OTELO; we have focused on the search of those AGNs. This is not indicative of a redshift preference but rather a selection effect. It should also be noted that the proportion of AGNs over the total sample of objects is higher at higher redshifts. This is expected since AGNs are very luminous objects and thus can be easily detected at higher redshifts.

Figure 7 represents the normalised distribution of magnitudes in the $r$ band, comparing the whole sample of OTELO with the selected emitters and the AGNs. As can be seen, the total and the ELC population show very similar distributions. Their median magnitudes are 26.4 and $26.0 \pm 2.2$, respectively. In the case of the AGN population, the distribution peaks at brighter magnitudes, the median being $24.5 \pm 2.0$. This result was expected since the AGN phenomenon usually occurs in galaxies with higher luminosities than those with pure stellar formation (Osterbrock 1991).

Below we summarise some of the characteristics of each AGN group according to their selection method.

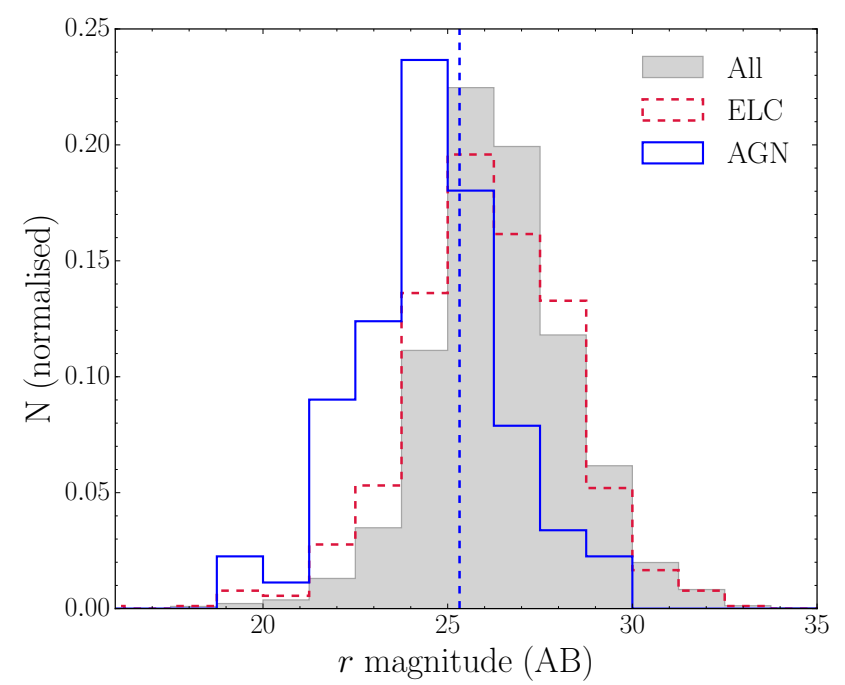

Fig. 7. Normalised magnitude distribution in the $r$ band of all the objects in the OTELO catalogue (grey), the selected emitters (red dashed line), and the population of AGNs (blue solid line). The AGN distribution peaks at $24.5 \mathrm{mag}$, one magnitude and a half brighter that the two other distributions.

\subsubsection{X-ray-detected AGNs}

X-ray surveys are an efficient method to select AGNs, as can be deduced from Table 2. A fraction of $43 \%$ of our AGNs (31) were selected exclusively using the X/O ratio described in Sect. 2.2. In total, this method selected $58 \%$ of the whole sample of AGNs (42). Moreover, of the 52 sources with $\mathrm{X} / \mathrm{O}$ information, $81 \%$ turned out to be AGNs, thus signaling that active galaxies could be responsible for the majority of the X-ray emission.

X-ray-selected AGNs can be divided into two groups according to their level of obscuration caused by large columns of gas along the line of sight $\left(N_{\mathrm{H}}>10^{22} \mathrm{~cm}^{-2}\right)$. In order to distinguish between unobscured and obscured X-rays AGNs, we used the hardness ratio, defined by Pović et al. (2009) as follows:

$\operatorname{HR}\left(\Delta E_{1} / \Delta E_{2}\right)=\frac{\mathrm{CR}\left(\Delta E_{1}\right)-\mathrm{CR}\left(\Delta E_{2}\right)}{\operatorname{CR}\left(\Delta E_{1}\right)+\operatorname{CR}\left(\Delta E_{2}\right)}$,

where $\Delta E_{1}$ and $\Delta E_{2}$ are two different energy bands, in our case $\Delta E_{1}=2-4.5 \mathrm{keV}$ (hard2 band) and $\Delta E_{2}=0.5-2 \mathrm{keV}$ (soft band), and $\operatorname{CR}\left(\Delta E_{\mathrm{n}}\right)$ is the count rate in the corresponding band. We used the criterion by Della Ceca et al. (2004), who found that $90 \%$ of their type 1 AGNs fell inside a narrow limit: $-0.75<\mathrm{HR}<-0.35$, while type 2 sources occupied a broader range with $\mathrm{HR}>-0.35$.

In total, 21 out of our $42 \mathrm{X}$-ray-selected AGNs included the hardness ratio information in their catalogue listing. With the method described above, 15 sources were selected in the first category and 6 in the second. This represents a fraction of $71 \%$ unobscured and $29 \%$ obscured X-ray AGNs over the total subsample of those objects possessing information of their hardness ratio. This is in agreement with what was found by Marchesi et al. (2016), where $69 \%$ and $31 \%$ of their whole sample of X-ray AGNs (both type 1 and type 2) were unobscured and obscured, respectively.

\subsubsection{Active galactic nuclei selected based on mid-infrared}

The MIR selection methods described in Sect. 2.3 effectively selected 29 AGNs, $40 \%$ of the sample. Eighteen of those objects, 


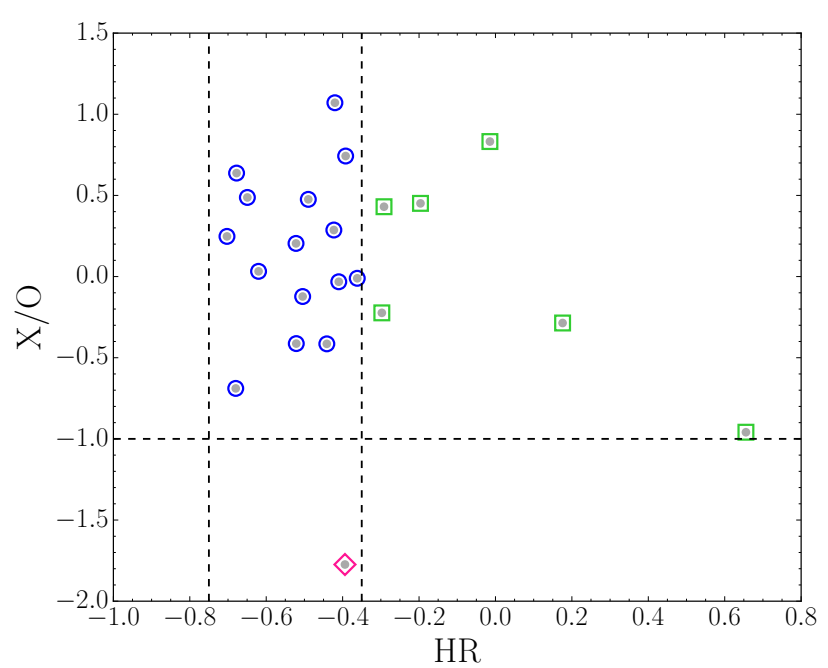

Fig. 8. X-ray-to-optical flux ratio $(\mathrm{X} / \mathrm{O})$ as a function of the hardness ratio, for the sources of Pović et al. (2009) (grey dots) in the OTELO catalogue. The horizontal line corresponds to the limit $\mathrm{X} / \mathrm{O}=-1$, which separates AGNs $(\mathrm{X} / \mathrm{O}>-1)$ from SFGs $(\mathrm{X} / \mathrm{O}<-1)$. The two vertical lines correspond to the limits set by Della Ceca et al. (2004) which encloses type 1 AGNs $(-0.75<\mathrm{HR}<-0.35)$, represented here by blue circles. Green squares are sources with HR $>-0.35$, i.e. type 2 AGNs. The pink diamond represents a probable non-AGN source, which could be a coronal emitting star, a star-forming or early-type galaxy, or a heavily absorbed (Compton thick) AGN.

that is, a quarter of the AGN sample, were not selected by any other method. This implies that MIR selection is the second most effective method to select AGNs in this work. However, the fraction of IR AGNs over the total IR population is relatively small. In fact, barely $1 \%$ of the objects for which information is available in the four IRAC bands were selected as AGNs using the revised IRAC criteria from Donley et al. (2012) (see Fig. 4). Similarly, of the objects for which information is available in the 4.5 and $8.0 \mu \mathrm{m}$ bands from IRAC and in the $24 \mu \mathrm{m}$ band from MIPS, only 3\% were classified as AGNs according to the KIM criteria of Messias et al. (2012). The striking difference in the number of AGNs versus the total population of X-rays and MIR sources is also seen in the work of Cowley et al. (2016), who performed a similar multiwavelength AGN selection (compare their Figs. 3 and 4).

As already mentioned, the great advantage of MIR selection is that it allows us to detect even those AGNs that are heavily obscured in X-rays. Consequently, by comparing the objects selected with MIR and X-rays methods, we can determine the fraction of obscured AGNs whose X-ray emission has been heavily absorbed by the surrounding interstellar gas or dust and re-emitted at IR wavelengths. In our case, 11 objects were selected both with MIR and X-rays methods while 18 were only selected with the former. This implies that $38 \%$ of our IR AGNs are unobscured or moderately obscured and the rest (62\%) are heavily obscured. In their work, Mateos et al. (2012) selected AGNs with IR methods over the BUXS ${ }^{2}$ field and found that $38.5 \%$ had an X-ray counterpart, meaning they were not heavily obscured. This is in agreement with our findings.

\subsubsection{AGNs at $z \sim 0.4$}

Our final sample of $\mathrm{H} \alpha$ emitters at $z \sim 0.4$ was composed of 46 objects. From those, 12 were optically selected as AGNs (half

\footnotetext{
2 Bright Ultra-hard XMM-Newton Survey.
}

Table 3. OTELO sources at $z \sim 0.4$ and fraction of emitters and AGNs.

\begin{tabular}{lcccc}
\hline \hline $\begin{array}{l}\text { Objects at } \\
z \sim 0.4\end{array}$ & $\begin{array}{c}\mathrm{H} \alpha \\
\text { Emitters }\end{array}$ & Optical & $\begin{array}{c}\text { XGNs } \\
\text { X-rays }\end{array}$ & Total \\
\hline$\sim 186$ & 46 & 12 & 1 & 13 \\
$100 \%$ & $\sim 25 \%$ & & & $\sim 7 \%$ \\
& $100 \%$ & $26 \%$ & & \\
\hline
\end{tabular}

Notes. First column: total number of OTELO sources at $z \sim 0.4$ (see text for details). Second column: total number of $\mathrm{H} \alpha$ selected emitters. Third and fourth columns: number of optically selected and X-rays-selected AGNs at that redshift. Fifth column: total number of AGNs at that redshift. The second row shows the proportion of emitters and AGNs over the total sample of objects at that redshift. The third row indicates the proportion of optically selected AGNs (NLAGNs or BLAGNs) over the sample of emitters. Due to the small numbers that are being managed here, and the uncertainty in the estimation of the total number of sources at $z \sim 0.4$, these numbers, especially those in the second row, should be taken with caution.

of them being BLAGNs and half NLAGNs). From the rest of the AGN sample, only one object (X-ray-selected) fell at that redshift. In total, we have 13 AGNs at $z \sim 0.4$.

In order to evaluate the proportion of line emitters and AGNs at that redshift, we first estimated the total number of objects found at $z \sim 0.4$ in OTELO. Considering an error of $\sim 0.2$ in the redshifts calculated with LePhare, as indicated in Sect. 2.1, we focused our search on the spectral window $0.37<z<0.42$, which covers the $\mathrm{H} \alpha$ and [NII] lines in OTELO of \pm 0.2 in redshift. To these objects, we added the $\mathrm{H} \alpha$ emitters that did not have a redshift in that interval but were classified as $z \sim 0.4$ emitters by alternative methods. We avoided stars by discarding bright objects (with an $\mathrm{AB}$ magnitude in the deep image <24) with a stellarity index $>0.95$ from SExtractor. In total, the population of sources at $z \sim 0.4$ in OTELO was estimated to be approximately 186 objects. This would imply that $\sim 25 \%$ of the objects at $z \sim 0.4$ are line emitters, while $\sim 7 \%$ are AGNs. However, due to the small sample size here, these values may not be statistically significant. Furthermore, the fraction of optically selected AGNs (NLAGNs or BLAGNs) over the sample of $\mathrm{H} \alpha$ emitters is $26 \%$ (see Table 3 ).

While the total number of sources at $z \sim 0.4$ may be subject to errors due to the uncertainty in our photo- $z$ calculations, especially for the faintest sources, the sample of $\mathrm{H} \alpha$ emitters on the other hand was carefully inspected by different collaborators and therefore we fully rely on them. In their recent work from the HSC-SSP ${ }^{3}$, Hayashi et al. (2018) found $14513 \mathrm{H} \alpha$ emitters in a total comoving volume of $9.77 \times 10^{5} \mathrm{Mpc}^{3}$, using the NB921filter to select the objects. This volume is 508 times greater than that covered by the OTELO field in the redshift range $0.37<z<0.42$, which is $1924.31 \mathrm{Mpc}^{3}$. According to these results, we would expect to find $\sim 29 \mathrm{H} \alpha$ emitters in our field. This means we have found significantly (one third) more emitters in the OTELO survey. This difference may be attributed to the limiting line flux reached by the Subaru team $\left(1.5 \times 10^{-17} \mathrm{erg} \mathrm{s}^{-1} \mathrm{~cm}^{-2}\right)$, which is higher than ours $(\sim 1.6 \times$ $10^{-18} \mathrm{erg} \mathrm{s}^{-1} \mathrm{~cm}^{-2}$, see Fig. 9), and also to the fact that their detection method, based only on a colour-colour diagram, is less efficient. As a matter of fact, with this method they are only able to select objects with an observed EW > $25 \AA$ for the NB921 filter, while our restrictions in $\mathrm{EW}$ go much lower.

\footnotetext{
3 Hyper Suprime-Cam (HSC) Subaru Strategic Program (SSP). See http://hsc.mtk.nao.ac.jp/ssp/
} 


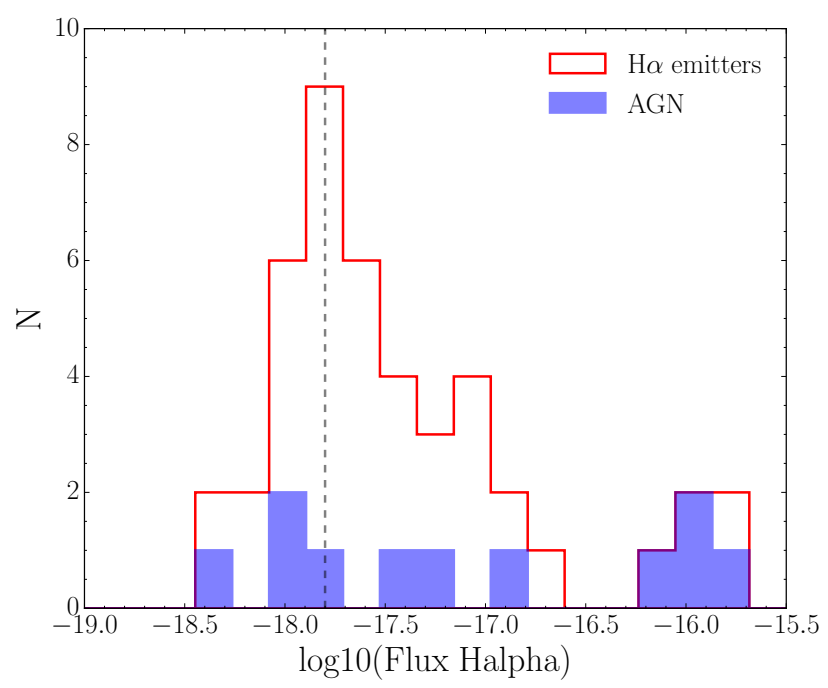

Fig. 9. Histogram of $\mathrm{H} \alpha$ fluxes of the sources selected as $\mathrm{H} \alpha$ emitters (red line) and the optically selected AGNs at $z \sim 0.4$ (blue). The grey dashed line marks the peak of the distribution, corresponding to a flux of $\sim 1.6 \times 10^{-18} \mathrm{erg} \mathrm{s}^{-1} \mathrm{~cm}^{-2}$.

Sobral et al. (2013) also conducted a survey to find $\mathrm{H} \alpha$ emitters at $z \sim 0.4$ using the NB921 filter and the same colour-colour diagram technique with an identical EW cut to that of the Subaru team. Sobral et al. (2013) found 1742 emitters over a cosmic volume of $8.8 \times 10^{4} \mathrm{Mpc}^{3}, 46$ times bigger than our own. Translated to the OTELO volume, this would imply 38 emitters in our field, a value closer to what we find but still smaller. It is clear from these two comparisons that the potential of OTELO's pseudo-spectra to select emitters is noticeable.

As for the AGN fraction, we found that Sobral et al. (2013) and other authors estimated an AGN contribution to the $\mathrm{H} \alpha$ population of $\sim 10-15 \%$, up to $z \sim 1$. This range is consistent with the results obtained from the analysis of emission-line galaxies at $z<0.36$ from SDSS and GAMA surveys performed by Lara-López et al. (2013), who set this contribution to $\sim 11 \%$ in each case. However, in a more recent work, Sobral et al. (2016) found that the AGN fraction strongly correlates with $\mathrm{H} \alpha$ luminosity. While for low luminosities the previous estimation is acceptable, for higher luminosities the AGN fraction strongly increases. These latter authors estimated the AGN fraction to be $30 \%$ and found that the most luminous $\mathrm{H} \alpha$ emitters at any cosmic time are BLAGNs. In our case, we find a higher mean proportion of optically selected AGNs (26\% of the overall $\mathrm{H} \alpha$ population, at any luminosity) although given our small numbers this is within the uncertainties (see Table 3). On the other hand, our fraction of AGNs is almost $100 \%$ at the highest luminosities, as shown in Fig. 9, in agreement with Sobral et al. (2016). Moreover, our brightest AGNs are the broad-line ones, as also found by these latter-mentioned authors.

\subsection{Morphology}

We studied the morphology of our AGNs using GALAPAGOS (Barden et al. 2012), a fully automated piece of software which combines the detection of objects with SExtractor and their light profile modelling with GALFIT (Peng et al. 2002). GALAPAGOS was run over the high-resolution images from the Hubble Space Telescope (F606W and F814W filters) corresponding to the OTELO field of view. The HST images cover the whole field of OTELO except for a $\sim 3.1 \mathrm{arcmin}^{2}$ region in the lower left-hand corner. The objects detected in this way were matched to the sources in the OTELO catalogue. GALFIT then obtained a light model with a Sérsic profile (Sérsic 1963) for each of the detected components, starting by the brightest ones.

This model was then subtracted to the original image in order to obtain a residual image, showing possible hidden subcomponents of the object. Several examples of this procedure (original HST images used for the detection, Sérsic profile modelled by GALFIT and residual images) are shown in Appendix B.

Of the total sample of 72 AGNs, a GALFIT Sérsic model with one or more components was obtained for 56 OTELO objects (and for their detected components in the high-resolution images). For the rest of them, either the source was so dim that it could not be fitted, or no HST image was available. We performed a visual classification of those objects by four collaborators based on the following parameters: (1) the appearance of the object in the HST images; (2) the GALFIT model and, in particular the value of the Sérsic index of the main component, $n$; (3) the existence (or not) of a residual after subtracting the model from the original image (revealing possible spiral arms, bars, and hidden structures); and (4) the relative colour of the source in a colour-colour diagram (such as $u-K_{\mathrm{s}}$ vs. $z-K_{\mathrm{s}}$, for instance). Based on that, each object was classed in one of the following categories: (1) point-like sources, (2) early-type sources (spheroidal objects, including ellipticals, E, and lenticulars, S0), (3) late-type sources (objects with disc, including spirals, S, and irregulars, Irr), and (4) unclassifiable objects. A summary of this classification is shown in Table 4.

The majority of our AGNs were classified as late-type objects $(64.3 \%)$. Of those, 12 were clearly spirals $(21 \%)$ such as the ones shown in Figs. B.1 and B.2, and 9 were irregulars $(16 \%)$ (see, e.g. Fig. B.13). On the other hand, $14.3 \%$ of our AGNs were classified as early-type objects, including two possible lenticulars such as the one shown in Fig. B.9. Finally, 12.5\% of the sample were point-like sources and thus possible QSOs (see, e.g. the objects in Figs. B.8 and B.11) and 8.9\% could not be classified (such as the one in Fig. B.4). In addition to that, 9 of the AGNs were flagged as multiple objects (16\%), meaning that what was seen as a single object in OTELO was actually a system of multiple components as revealed by the HST images (see Fig. B.3), and 9 (16\%) were flagged as having possible interactions or mergers (see Fig. B.6 or B.12).

If we study the morphological classification of our AGNs according to the selection methods, we can see that $80 \%$ of the BLAGNs at $z \sim 0.4$ are in spiral galaxies, while the only NLAGN that was classified at that same redshift is an earlytype instead. On the other hand, X-ray- and MIR-selected AGNs seem to share similar morphologies, although the fraction of late-type galaxies among the X-ray selected AGNs (56\%) is smaller than among the MIR ones (79\%). This is in agreement with Griffith \& Stern (2010), who found that their MIR-selected AGNs had a slightly higher incidence of being hosted by disc galaxies than the X-ray selected ones, although both had similar morphologies in general. These latter authors explained this according to the scenario proposed by Gabor et al. (2009), where AGNs represent an intermediate stage between disc-dominated and bulge-dominated galaxies. Hickox et al. (2009) also found results in agreement with this evolutionary scenario, where galaxies evolve from blue, disc-dominated types with radiatively efficient AGNs (optical- and IR-bright) to red, bulge-dominated ones with less efficient AGNs (optically faint, radio-bright) following the growth of the stellar bulge and a quasar phase. In this context, AGNs tend to be selected in MIR when the accretion to the SMBH is more effective and the reprocessing of UV 
Table 4. Morphological classification of OTELO AGNs.

\begin{tabular}{lcccccc}
\hline \hline & X-rays & $\begin{array}{c}\text { X-rays + MIR } \\
\text { (unobscured) }\end{array}$ & $\begin{array}{c}\text { MIR } \\
\text { (obscured) }\end{array}$ & $\begin{array}{c}\text { BLAGNs } \\
\text { at } z \sim 0.4\end{array}$ & $\begin{array}{c}\text { NLAGNs } \\
\text { at } z \sim 0.4\end{array}$ & Total \\
\hline Point-like & 3 & 2 & 2 & 0 & 0 & $\mathbf{7}$ \\
Early-type & 5 & 2 & 0 & 0 & 1 & $\mathbf{8}$ \\
Late-type & 14 & 6 & 11 & 5 & 0 & $\mathbf{3 6}$ \\
Unclassifiable & 3 & 0 & 1 & 0 & 1 & $\mathbf{5}$ \\
Total & $\mathbf{2 5}$ & $\mathbf{1 0}$ & $\mathbf{1 4}$ & $\mathbf{5}$ & $\mathbf{2}$ & $\mathbf{5 6}$ \\
\hline
\end{tabular}

Notes. The different types of AGNs, according to their selection method (X-rays, MIR, or BLAGNs/NLAGN at $z \sim 0.4$ ) are divided into four morphological categories: point-like sources, early-type (including ellipticals and lenticulars), late-type (including spirals and irregulars), and unclassifiable galaxies.

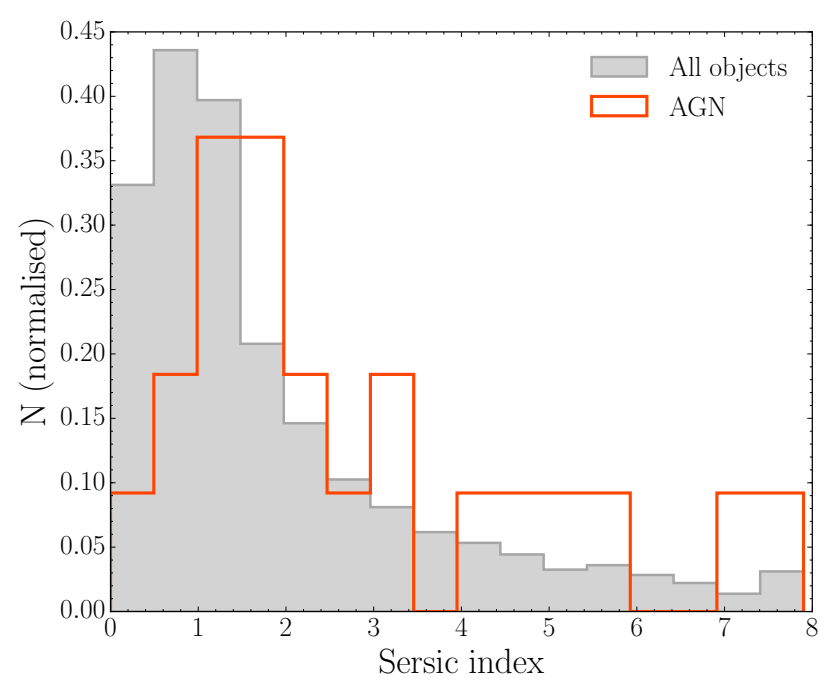

Fig. 10. Sersic indices from the GALFIT models obtained for objects with unique components. The grey distribution represents the whole sample of OTELO sources that have been modelled as a single component (3286 objects). The orange distribution represents the AGN subpopulation.

photons to MIR by the dust torus is significant, while they are better selected in X-rays when the accretion is less efficient.

We also analysed the distribution of Sérsic indices obtained with the GALFIT models. According to the equation of a Sérsic profile, higher indices imply more concentrated objects with a steeper decrement in brightness. For that purpose, we used only the objects matched with the OTELO catalogue as individual sources and discarded the multiple ones, which would require a deeper and more detailed analysis. In Fig. 10 the distribution of Sérsic indices is shown, both for the whole sample of OTELO objects and for the AGN subpopulation. For the total population of OTELO objects, the distribution peaks at $n \sim 1$ and decreases steadily up to the maximum ${ }^{4}$ value of $n=8$. The AGN distribution peaks at slightly higher indices and seems to have a higher proportion of concentrated objects. However, due to the small numbers being managed in the AGN sample, these variations may be attributed to statistical differences. As a matter of fact, Fan et al. (2014) performed a similar analysis over a sample of X-ray-selected AGNs at $z \sim 2$ and found very similar AGN and non-AGN distributions to ours (see their Fig. 2). Their numbers were also small (35 AGNs) and they concluded that there was no statistical difference between the AGNs and the control sample.

\footnotetext{
4 The minimum and maximum constraints set in GALAPAGOS/GALFIT were 0.2 and 8 , respectively.
}

In any case, the distribution of Sérsic indices agrees with our previous findings about the predominance of late-type galaxies with a disc among the AGNs.

\subsection{Luminous and ultra-luminous infrared galaxies}

Luminous and ultra-luminous infrared galaxies are among the brightest galaxies in the Universe. As their names suggest, they emit most of their radiation in the IR, their luminosity in this range being superior to $10^{11}$ and $10^{12} L_{\odot}$, respectively. The power source responsible for this emission is believed to be a starburst and/or an AGN. In order to look for these objects in OTELO, we first derived the IR luminosity $\left(L_{\mathrm{IR}}\right)$ of our sources. This luminosity is defined as the emission in the spectral range from 8 to $1000 \mu \mathrm{m}$. In this work, we took advantage of our multiwavelength catalogue and the fact that our sources may have not only MIPS photometry but also fluxes in the 100 and $160 \mu \mathrm{m}$ Herschel/PACS bands and in the 250, 350, and $500 \mu \mathrm{m}$ bands from Herschel/SPIRE. We used the IR luminosity calculated by LePhare, which achieves this by integrating the emission in the range $8-1000 \mu \mathrm{m}$ from the best FIR SED fitted to each galaxy.

The distributions of IR luminosities obtained in this way are shown in Fig. 11. As can be seen, at higher luminosities the proportion of AGNs contributing to the total $L_{\mathrm{IR}}$ is greater. We can also deduce from the figure that the proportion of LIRGs and ULIRGs among AGNs is significant. However, in order to draw conclusions, we need to estimate the redshift up to which the sample of LIRGs and ULIRGs is complete. To do so, we used the minimum $24 \mu \mathrm{m}$ MIPS flux detected in OTELO (21.95 AB) and a FIR SED template from Chary \& Elbaz (2001). The template was redshifted from $z=0$ to 2.5 and re-escalated so that the minimum flux would correspond to the MIPS photometric point. Subsequently, the IR luminosity was obtained by integrating the SED flux from 8 to $1000 \mu \mathrm{m}$. The results are shown in Fig. 12. As expected (see, e.g. Fig. 4 from Elbaz et al. 2011), the minimum detectable $L_{\mathrm{IR}}$ increases with redshift. Given our sensitivity limits, we are able to detect all the LIRGs up to $z \sim 1.6$, while the ULIRGs sample is complete at all redshifts.

The distribution of all the LIRGs and ULIRGs found in OTELO, as a function of redshift, is shown in Fig. 13. It can be seen that the number of LIRGs is much higher than that of ULIRGs at low redshifts, and that it increases with $z$ (as also found by Magnelli et al. 2013, see its Fig. 12) up to $z \sim 1.5$, from where it starts to decrease as a consequence of our detection limits and in agreement with the previous estimation. At high redshifts, on the other hand, the number of ULIRGs prevails. Our distributions of LIRGs and ULIRGs over redshift are in agreement with what was found by Lin et al. (2016) and Małek et al. (2017). 


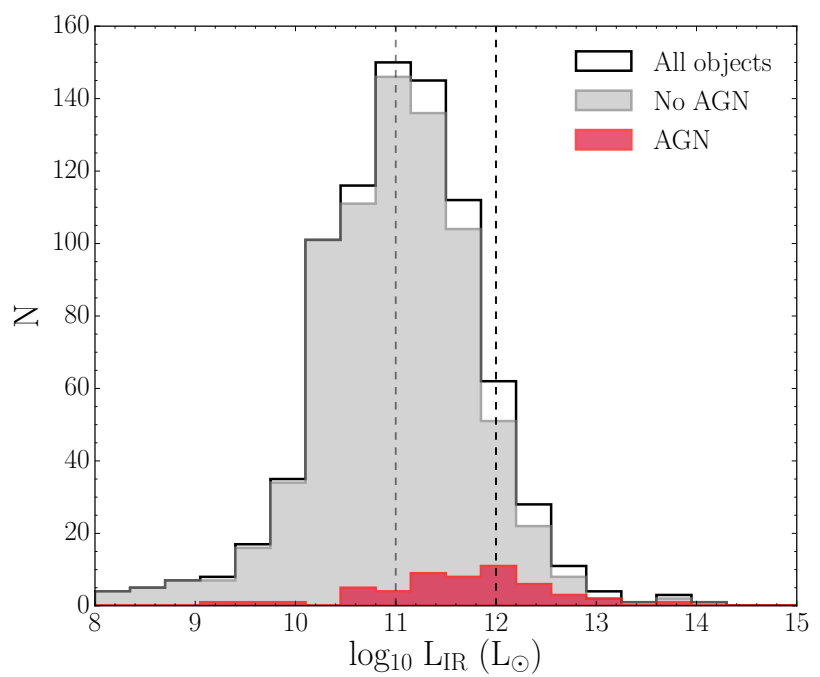

Fig. 11. Distribution of IR luminosities for the whole sample of OTELO sources for which $24 \mu \mathrm{m}$ photometry is available (black solid line), the objects not detected as AGNs (grey), and the selected AGNs (red). The grey and black dashed lines indicate the LIRGs and ULIRGs limit, respectively: $10^{11}$ and $10^{12} L_{\odot}$.

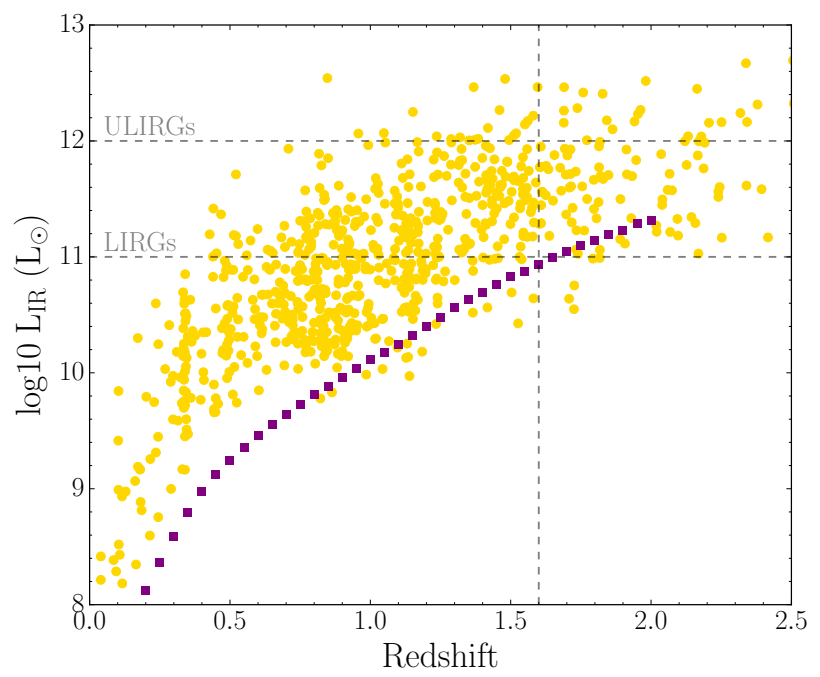

Fig. 12. Infrared luminosity in OTELO as a function of redshift. Yellow dots represent the IR luminosity of OTELO sources for which $24 \mu \mathrm{m}$ photometry is available. Purple squares represent the minimum detectable $L_{\mathrm{IR}}$ given the sensitivity limits in our catalogue (see text for details). Horizontal lines indicate the LIRGs and ULIRGs limits. The vertical line shows the redshift up to which the sample of LIRGs is complete in our survey $(z=1.6)$.

Table 5 details the number of ULIRGs found over the total sample of OTELO objects with $L_{\mathrm{IR}}$, as well as among the AGN and non-AGN galaxies. We found that the fraction of LIRGs and ULIRGs is higher among the AGN population than among the rest of the galaxies, and that this difference is specially remarkable for the ULIRGs: $57 \%$ (40\%) of AGNs (non-AGNs) are LIRGs, while $33 \%$ (8\%) are ULIRGs.

We also found that $8 \%$ of LIRGs up to $z=1.6$ are active galaxies and that this number increases to $22 \%$ for ULIRGs. This result is in agreement with Małek et al. (2017), who found that ULIRGs are characterised by a higher fraction of AGNs than LIRGs. Among the 17 ULIRGs that are AGNs, all but one (21\%) were selected with MIR methods. This coincides with what was

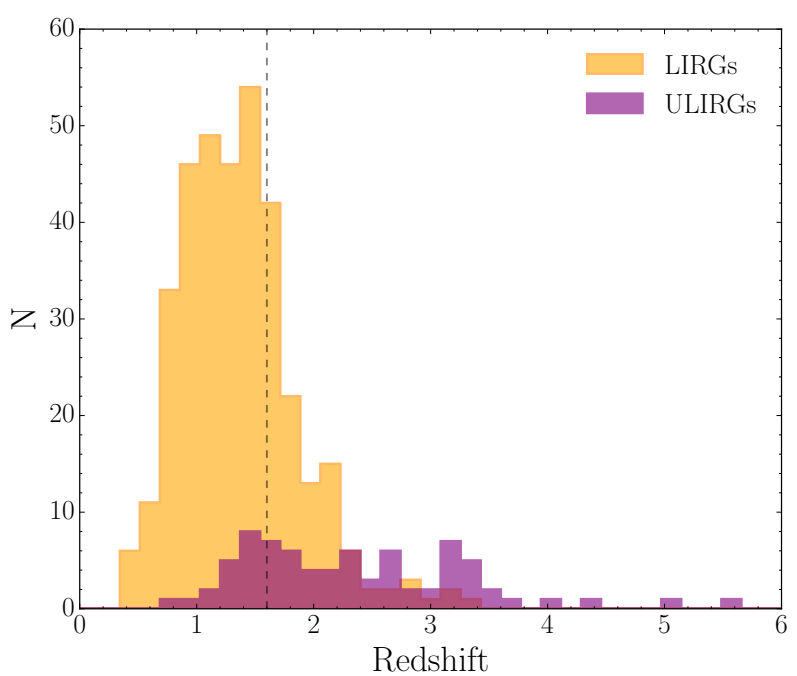

Fig. 13. Redshift distribution for LIRGs and ULIRGs (orange and purple, respectively). The grey dashed line indicates $z=1.6$, the redshift up to which the sample of LIRGs is complete.

Table 5. Number of LIRGs and ULIRGs found among the AGN and non-AGN populations, as well as the whole sample of OTELO sources with $L_{\mathrm{IR}}$.

\begin{tabular}{lrrr}
\hline \hline & $\begin{array}{r}\text { LIRGs }^{(*)} \\
(z<1.6)\end{array}$ & $\begin{array}{r}\text { ULIRGs } \\
(z<1.6)\end{array}$ & $\begin{array}{r}\text { ULIRGs } \\
(\text { all } z)\end{array}$ \\
\hline AGNs & $21(57 \%)$ & $6(16 \%)$ & $17(33 \%)$ \\
non-AGNs & $243(39 \%)$ & $15(2.4 \%)$ & $60(7.9 \%)$ \\
Total & $264(40 \%)$ & $21(3.2 \%)$ & $77(9.5 \%)$ \\
\hline
\end{tabular}

Notes. The numbers in parentheses represent the fraction of each kind of object over the given population. ${ }^{(*)}$ The LIRGs sample is studied only up to $z=1.6$ for completeness purposes (see text for details), while the ULIRGs sample covers all redshifts.

found by Veilleux et al. (1997), who claimed that 25-30\% of their ULIRGs showed signs of activity in the optical or NIR.

\subsection{AGN environment at $z \sim 0.4$}

Following a general analysis of the AGNs found in OTELO, we now focus on the population of objects at $z \sim 0.4$. We studied the environments of AGNs at this redshift in an attempt to determine whether they tend to be in high- or low-density environments. For details of the luminosity function (LF) of all sources of this population in the OTELO field, as well as the contribution of the AGN hosts to this LF, we refer the reader to Ramón-Pérez et al. (2019).

The role of environment in AGN triggering is one of the most important open questions in the field. Both the large-scale and the local environments seem to be decisive in the evolution and properties of galaxies. In this section, we attempt to study the environments of AGNs at $z \sim 0.4$. The spatial distribution of all the sources at this redshift is shown in Fig. 14, along with that of the $\mathrm{H} \alpha$ emitters and AGNs.

We studied the surface density by means of the projected fifthnearest-neighbour distance $\left(D_{5}\right)$ of each source. This method provides the most accurate estimate of local galaxy density when compared to other techniques such as the use of counts in a fixed aperture or the Voronoi volume, according to Cooper et al. (2005). We used the edge correction by Kovač et al. (2010) to 


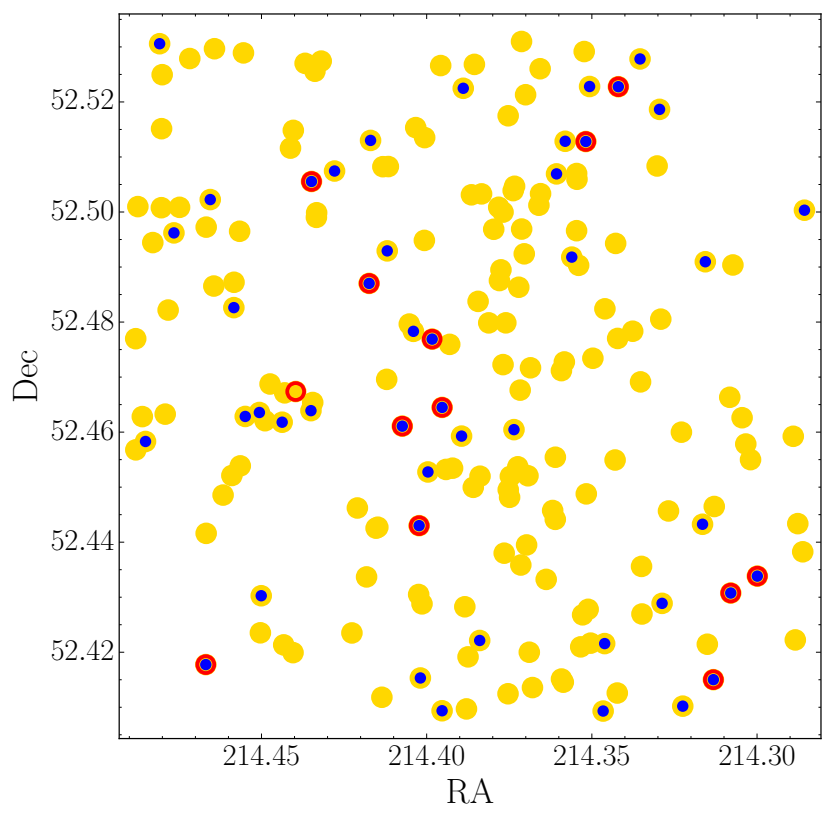

Fig. 14. Distribution of sources at $z \sim 0.4$ in OTELO. Filled yellow circles represent all the sources at that redshift. Filled blue circles are the $\mathrm{H} \alpha$ emitters, while red circles are the selected AGNs.

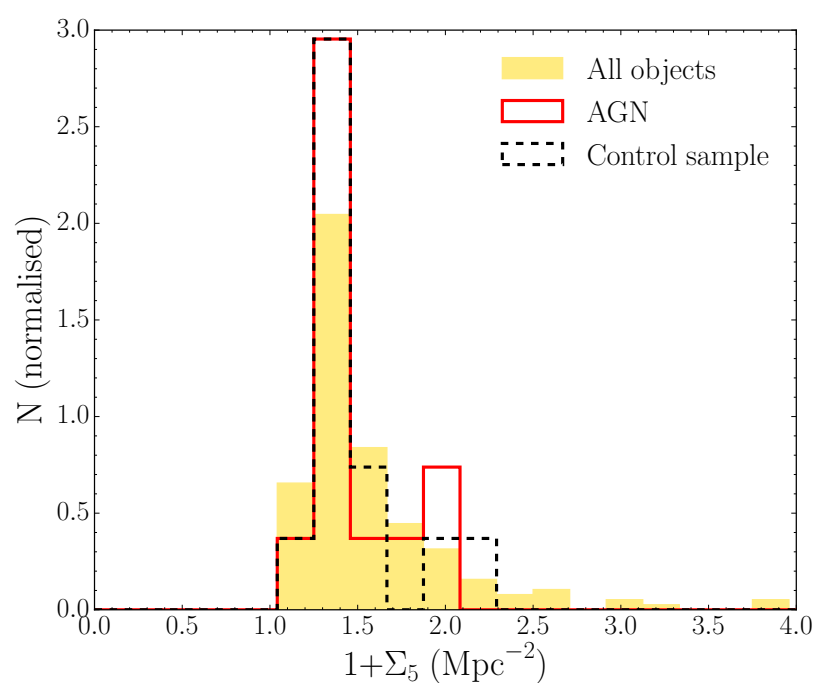

Fig. 15. Distribution of the surface density parameter $\left(\Sigma_{5}\right)$ for the whole sample of OTELO objects at $z \sim 0.4$ (yellow), the AGNs (red) and a random control sample of non-AGNs (black).

avoid obtaining artificially lower densities in the regions close to the edges of the field. The surface density is calculated as

$\Sigma_{5}=\frac{5}{\pi D_{5}^{2}}$.

We compared the distributions of the surface-density parameter for the sample of AGNs at $z \sim 0.4$ (13 objects) and for a control sample of 13 non-AGNs randomly chosen at the same redshift. As can be seen in Fig. 15, galaxies tend to concentrate in low-density environments. The AGN and non-AGN distributions are very similar, and seem to be in agreement with the density distribution of the overall population. We performed the analysis for different control samples and obtained analogous results every time.

To further analyse the environmental differences between the AGNs and the control sample, we looked at the distance

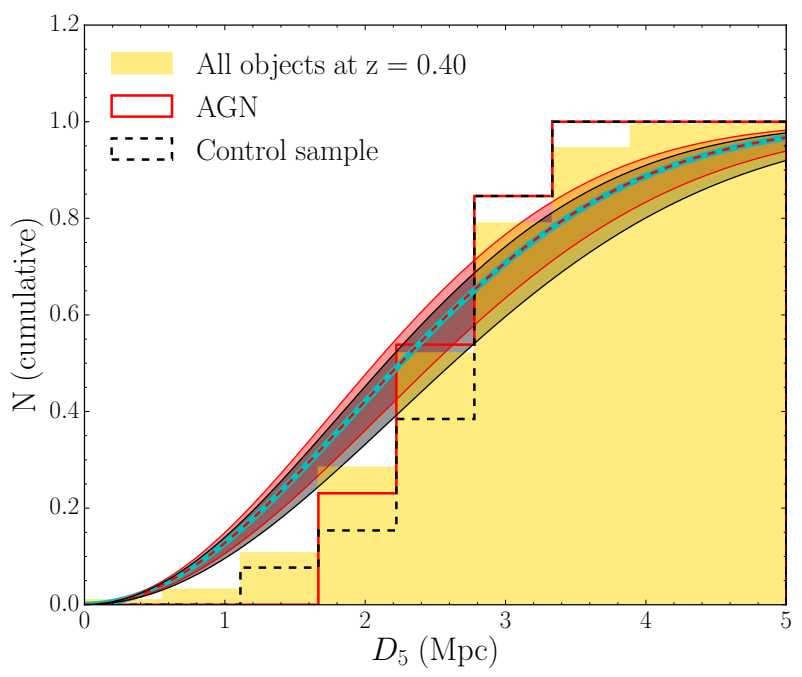

Fig. 16. Cumulative and normalised distributions of $D_{5}$ for the total sample of OTELO objects, the AGNs, and the control sample (nonAGNs). The yellow distribution is the distribution of all the objects at $z \sim 0.4$ and the cyan solid line is its Poissonian fit. The step histograms in red and black represent the distributions of $D_{5}$ for the AGN and nonAGN samples, respectively, while the corresponding dashed lines are their Poissonian fits. The filled bands show the propagation of statistical errors in the Poissonian fit.

parameter, $D_{5}$, in each sample. The distance from a randomly chosen source to the fifth-nearest other object follows a homogenous Poisson process (Martínez \& Saar 2002) whose probability distribution function in $2 \mathrm{D}$ is given by

$G\left(D_{5}\right)=1-\exp \left(-\rho \pi D_{5}^{2}\right)$

where $\rho$ is the intensity of the process, or the expected value of $D_{5}$. We fitted the cumulative distributions of $D_{5}$ in each sample to this function, assuming statistical errors of Poissonian nature, and compared it to the distribution of the overall population, composed of all the objects at $z \sim 0.4$. The results are shown in Fig. 16. It is clear from this figure that the AGN population and the control group do not statistically differ. This indicates that AGNs in OTELO field at $z \sim 0.4$ are found in identical density environments to non-AGNs.

\section{Conclusions}

This work has focused on the identification and characterisation of AGNs in the OTELO survey, an emission-line object survey that uses the red tunable filter of the OSIRIS instrument at the GTC (Bongiovanni et al.2019). We have obtained a sample of 72 AGNs in the OTELO field, selected using four different methods: an optical diagnostic diagram based on the $\mathrm{H} \alpha+[\mathrm{NII}]$ fluxes of the emitters at $z \sim 0.4$, a selection of BLAGNs at the same redshift based on the width of the emission line as seen in the pseudo-spectra, an $\mathrm{X}$-ray selection, and two diagnostic diagrams in the MIR.

The main goal of this work is to identify the main properties of the selected AGN population in OTELO. A detailed study on their demography, morphology, IR luminosity, and environment has been conducted. The main results from this analysis are summarised in the following paragraphs.

Regarding the AGN selection, X-ray emission has demonstrated to be the most efficient method, as similarly found by Mushotzky (2004). This method selected 58\% of the whole sample of AGNs in OTELO. Around one third of these X-ray-selected AGNs are obscured. Mid-infrared diagnostic diagrams are also 
very effective, selecting $40 \%$ of the OTELO AGN sample. In this case, the proportion of obscured and unobscured AGNs is $62 \%$ and $38 \%$, respectively. This is roughly the opposite to what is found by selecting with X-rays. Both results are in agreement with previous works, such as Mateos et al. (2012) and Marchesi et al. (2016).

In the optical, at $z \sim 0.4$ we found up to 13 AGNs, which represent $26 \%$ of the $\mathrm{H} \alpha$ emitters at that redshift. Compared to Sobral et al. (2016), we found a higher mean proportion of optically selected AGNs. Following morphological criteria, the majority of our total sample of AGNs (64.3\%) were classified as late-type galaxies; this percentage includes a $16 \%$ fraction of irregulars. A $14.3 \%$ fraction were classified as early-type and $12.5 \%$ as point-like sources, while $8.9 \%$ could not be classified. Moreover, a $16 \%$ fraction of the total sample show signs of interactions or mergers. Furthermore, most of the BLAGNs at $z \sim 0.4$ are spiral galaxies (4 out of 5). Finally, the fraction of late-type galaxies among the X-ray-selected AGNs (56\%) is smaller than among the MIR-selected ones (79\%). This result is in agreement with Griffith \& Stern (2010) and the evolution scenario proposed by Gabor et al. (2009), where AGNs represent an intermediate stage between disc-dominated and bulge-dominated galaxies.

According to their IR luminosity, we are able to recover all the LIRGs in the OTELO field up to $z \sim 1.6$. As expected, the fraction of LIRGs and ULIRGs is higher among the AGN population than among the rest of the galaxies, and this difference is particularly remarkable for the ultra-luminous type. However, the population of ULIRGs contains a higher fraction of AGNs than that of the LIRGs. Similar results were found by other recent works such as Lin et al. (2016) and Małek et al. (2017). Active galactic nuclei in the OTELO field at $z \sim 0.4$ are found in identical environments as non-AGNs (in agreement with Virani et al. 2000 or Waskett et al. 2005) but the subpopulation of AGNs at $z \sim 0.4$ is the most clustered group when compared to passive galaxies, SFGs, and $\mathrm{H} \alpha$ emitters, something also observed by Manzer \& De Robertis (2014).

Acknowledgements. This work was supported by the Spanish Ministry of Economy and Competitiveness (MINECO) under the grants AYA2013-46724-P AYA2013 - 42227 - P, AYA2014 - 58861 - C3 - 1 - P, AYA2014 - 58861 - C3 - 2 - P, AYA2014 - 58861 - C3 - 3 - P, AYA2016 - 75808 - R, AYA2016 - 75931 - C2 - 1 P, AYA2016 - 75931 - C2 - 2 - P, AYA2016 - 76682C3 - 1 - P, AYA2017 - 88007 C3-1-P and AYA2017-88007-C3-2-P. The authors thank the anonymous referee for her/his feedback and suggestions. Based on observations made with the Gran Telescopio Canarias (GTC), installed in the Spanish Observatorio del Roque de los Muchachos of the Instituto de Astrofísica de Canarias, in the island of La Palma. MP acknowledges financial supports from the Ethiopian Space Science and Technology Institute (ESSTI) under the Ethiopian Ministry of Innovation and Technology (MInT), and from the State Agency for Research of the Spanish MCIU through the "Center of Excellence Severo Ochoa" award for the Instituto de Astrofísica de Andalucía (SEV-2017-0709). EJA acknowledges financial support from the State Agency for Research of the Spanish MCIU through the "Center of Excellence Severo Ochoa" award for the Instituto de Astrofísica de Andalucía (SEV-2017-0709).

\section{References}

Alexander, D. M., Brandt, W. N., Hornschemeier, A. E., et al. 2001, AJ, 122, 2156

Álvarez, P., Rodríguez Espinosa, J. M., \& Sánchez, F. 1998, New Astron. Rev., 42,553

Arribas, S., Mediavilla, E., García-Lorenzo, B., \& del Burgo, C. 1997, ApJ, 490, 227

Baldwin, J. A., Phillips, M. M., \& Terlevich, R. 1981, PASP, 93, 5

Barden, M., Häußler, B., Peng, C. Y., McIntosh, D. H., \& Guo, Y. 2012, MNRAS 422, 449

Barro, G., Pérez-González, P. G., Gallego, J., et al. 2011, ApJS, 193, 13

Blandford, R. D., Netzer, H., Woltjer, L., Courvoisier, T. J. L., \& Mayor, M. 1990, Active Galactic Nuclei, 97
Bongiovanni, A., Ramón-Pérez, M., Pérez García, A. M., et al. 2019, A\&A, 631, A9

Brandt, W. N., Alexander, D. M., Bauer, F. E., \& Vignali, C. 2004, in Physics of Active Galactic Nuclei at All Scales, eds. D. Alloin, R. Johnson, \& P. Lira (Berlin: Springer-Verlag) [arXiv:astro-ph/0403646]

Cepa, J., Alfaro, E. J., Bland-Hawthorn, J., et al. 2003, Rev. Mex. Astron. Astrofis. Conf. Ser., 16, 64

Chary, R., \& Elbaz, D. 2001, ApJ, 556, 562

Cid Fernandes, R., Stasińska, G., Schlickmann, M. S., et al. 2010, MNRAS, 403, 1036

Cid Fernandes, R., Stasińska, G., Mateus, A., \& Vale Asari, N. 2011, MNRAS, 413, 1687

Cooper, M. C., Newman, J. A., Madgwick, D. S., et al. 2005, ApJ, 634, 833

Cowley, M. J., Spitler, L. R., Tran, K.-V. H., et al. 2016, MNRAS, 457, 629

Della Ceca, R., Maccacaro, T., Caccianiga, A., et al. 2004, A\&A, 428, 383

Donley, J. L., Koekemoer, A. M., Brusa, M., et al. 2012, ApJ, 748, 142

Elbaz, D., Dickinson, M., Hwang, H. S., et al. 2011, A\&A, 533, A119

Fan, L., Fang, G., Chen, Y., et al. 2014, ApJ, 784, L9

Gabor, J. M., Impey, C. D., Jahnke, K., et al. 2009, ApJ, 691, 705

Griffith, R. L., \& Stern, D. 2010, ApJ, 140, 533

Hayashi, M., Tanaka, M., Shimakawa, R., et al. 2018, PASJ, 70, S17

Hickox, R. C., Jones, C., Forman, W. R., et al. 2009, ApJ, 696, 891

Ho, L. C., Filippenko, A. V., \& Sargent, W. L. W. 1997, ApJS, 112, 315

Hornschemeier, A. E., Brandt, W. N., Garmire, G. P., et al. 2001, ApJ, 554, 742

Kaspi, S., Maoz, D., Netzer, H., et al. 1996, ApJ, 470, 336

Kewley, L. J., Dopita, M. A., Sutherland, R. S., Heisler, C. A., \& Trevena, J. 2001, ApJ, 556, 121

Kewley, L. J., Groves, B., Kauffmann, G., \& Heckman, T. 2006, MNRAS, 372, 961

Kovač, K., Lilly, S. J., Cucciati, O., et al. 2010, ApJ, 708, 505

Lacy, M., Storrie-Lombardi, L. J., Sajina, A., et al. 2004, ApJS, 154, 166

Laird, E. S., Nandra, K., Georgakakis, A., et al. 2009, ApJS, 180, 102

Lara-López, M. A., Cepa, J., Castañeda, H., et al. 2011, PASP, 123, 252

Lara-López, M. A., Hopkins, A. M., López-Sánchez, A. R., et al. 2013, MNRAS, 434, 451

Lin, M.-Y., Hashimoto, Y., \& Foucaud, S. 2016, MNRAS, 456, 2735

Lutz, D., Poglitsch, A., Altieri, B., et al. 2011, A\&A, 532, A90

Maccacaro, T., Gioia, I. M., Wolter, A., Zamorani, G., \& Stocke, J. T. 1988, ApJ, 326, 680

Magnelli, B., Popesso, P., Berta, S., et al. 2013, A\&A, 553, A132

Małek, K., Bankowicz, M., Pollo, A., et al. 2017, A\&A, 598, A1

Manzer, L. H., \& De Robertis, M. M. 2014, ApJ, 788, 140

Marchesi, S., Lanzuisi, G., Civano, F., et al. 2016, ApJ, 830, 100

Martínez, V. J., \& Saar, E. 2002, Statistics of the Galaxy Distribution (Boca Raton: Published by Chapman \& Hall/CRC)

Mateos, S., Alonso-Herrero, A., Carrera, F. J., et al. 2012, MNRAS, 426, 3271

Messias, H., Afonso, J., Salvato, M., Mobasher, B., \& Hopkins, A. M. 2012, ApJ, 754, 120

Messias, H., Afonso, J. M., Salvato, M., Mobasher, B., \& Hopkins, A. M. 2014, A\&A, 562, A144

Morrissey, P., Conrow, T., Barlow, T. A., et al. 2007, ApJS, 173, 682

Mushotzky, R. 2004, Supermassive Black Holes in the Distant Universe, 308, 53

Osterbrock, D. E. 1991, Rep. Prog. Phys., 54, 579

Peng, C. Y., Ho, L. C., Impey, C. D., \& Rix, H.-W. 2002, ApJ, 124, 266

Planck Collaboration XX. 2016, A\&A, 594, A13

Pović, M., Sánchez-Portal, M., Pérez García, A. M., et al. 2009, ApJ, 706, 810

Ramón-Pérez, M., Bongiovanni, A., Pérez García, A. M., et al. 2019, A\&A, 631, A10

Roseboom, I. G., Oliver, S. J., Kunz, M., et al. 2010, MNRAS, 409, 48

Sánchez-Portal, M., Pintos-Castro, I., Pérez-Martínez, R., et al. 2015, A\&A, 578, A30

Sérsic, J. L. 1963, Boletín de la Asociación Argentina de Astronomía, La Plata, Argentina, 6, 41

Sobral, D., Smail, I., Best, P. N., et al. 2013, MNRAS, 428, 1128

Sobral, D., Kohn, S. A., Best, P. N., et al. 2016, MNRAS, 457, 1739

Stasińska, G., Cid Fernandes, R., Mateus, A., Sodré, L., \& Asari, N. V. 2006, MNRAS, 371, 972

Stern, D., Eisenhardt, P., Gorjian, V., et al. 2005, ApJ, 631, 163

Stocke, J. T., Morris, S. L., Gioia, I. M., et al. 1991, ApJS, 76, 813

Szokoly, G. P., Bergeron, J., Hasinger, G., et al. 2004, ApJS, 155, 271

Veilleux, S., \& Osterbrock, D. E. 1987, ApJS, 63, 295

Veilleux, S., Sanders, D. B., \& Kim, D.-C. 1997, ApJ, 484, 92

Virani, S. N., De Robertis, M. M., \& VanDalfsen, M. L. 2000, ApJ, 120, 1739

Waskett, T. J., Eales, S. A., Gear, W. K., et al. 2005, MNRAS, 363, 801 


\section{Appendix A: Broad-line AGNs at $z \sim 0.4$ in OTELO}
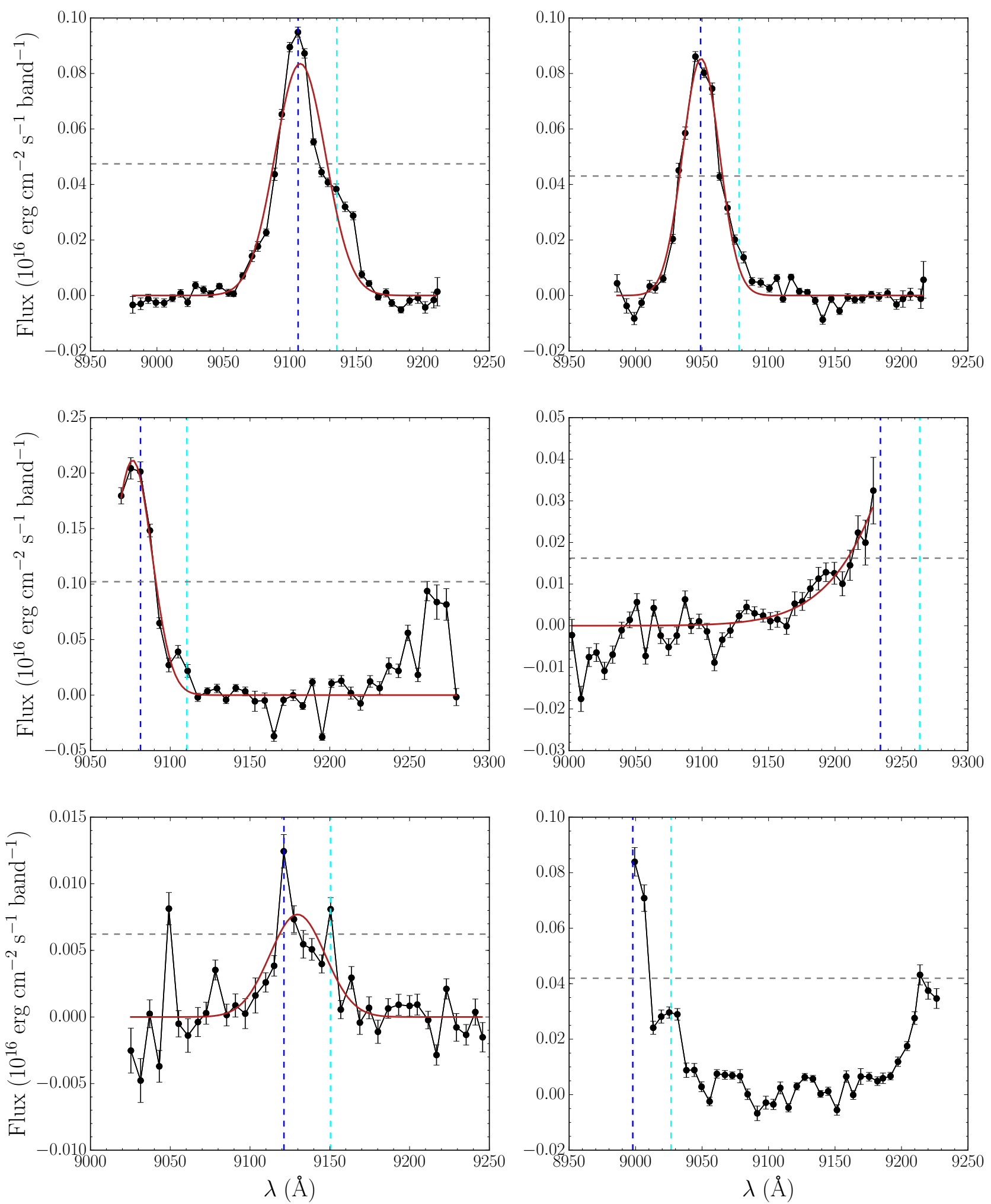

Fig. A.1. Objects from OTELO survey showing $\mathrm{H} \alpha+[\mathrm{NII}]$ emission and classified as BLAGNs. The red lines show the best fit to a Gaussian profile, while the grey dashed lines represent half the maximum value of the pseudo-spectra. The blue and cyan vertical lines mark the positions of the $\mathrm{H} \alpha$ and $[\mathrm{NII}]$ emission lines, respectively. In the last case, no fitting could be made due to the truncation of the line. However, the object was included in the final sample because its width is comparable to the rest of the objects selected as BLAGNs. 
M. Ramón-Pérez et al.: Active galactic nuclei in the OTELO survey

\section{Appendix B: AGN morphologies (examples)}

In the following pages of this appendix, the morphologies of some of the AGNs found in OTELO are shown as representative examples:

- Broad-line AGNs at $z \sim 0.4$ : Figs. B.1, B.2 and B.3.

- Narrow-line AGNs at $z \sim 0.4$ : Figs. B.4 and B.5.
- X-rays selected AGNs: Figs. B.6-B.9.

- Unobscured AGNs (MIR+X-rays selection): Figs. B.10 and B.11.

- Obscured AGNs (MIR selection only): Figs. B.12-B.15.

The figures display the object in the original HST images along with their GALFIT model and residual, both described in Sect. 3.2.
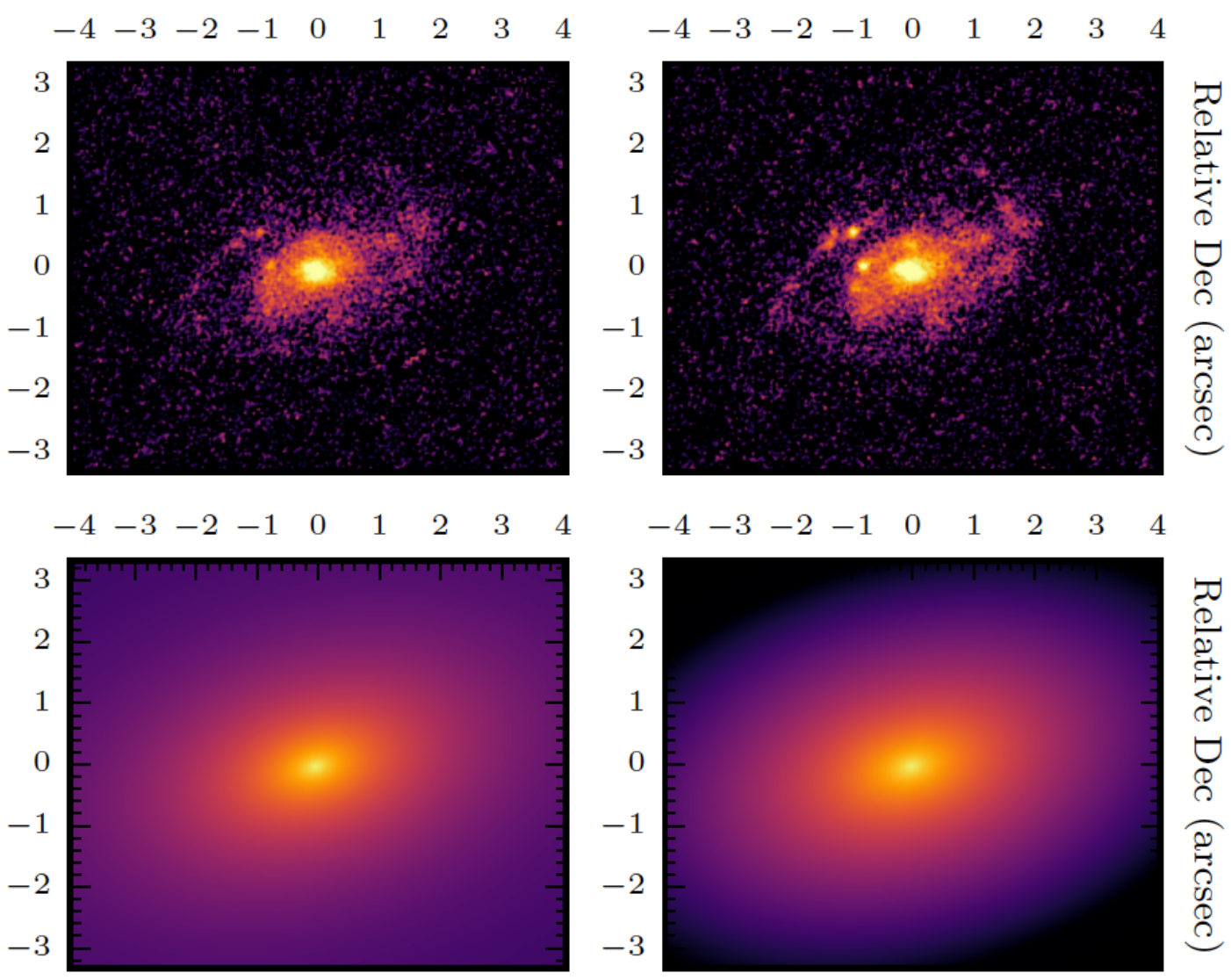

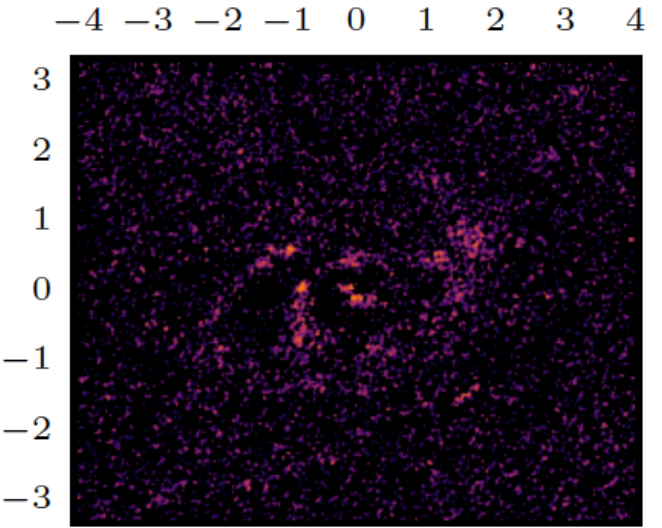

Relative RA (arcsec)

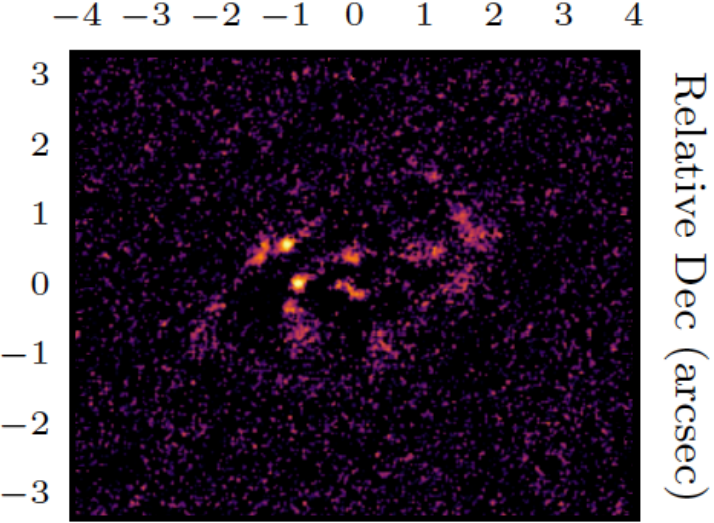

Relative RA (arcsec)

Fig. B.1. Morphology of the object \#1873. This object was classified as a BLAGNs at $z \sim 0.4$ and is clearly a spiral galaxy (S, late-type). The spiral arms are visible in the residual. First row: HST images of the object. Second row: GALFIT models. Third row: GALFIT residuals obtained by subtracting its model from the original image. First column: $V$ filter of HST (F814W) and second one: I filter (F606W). 

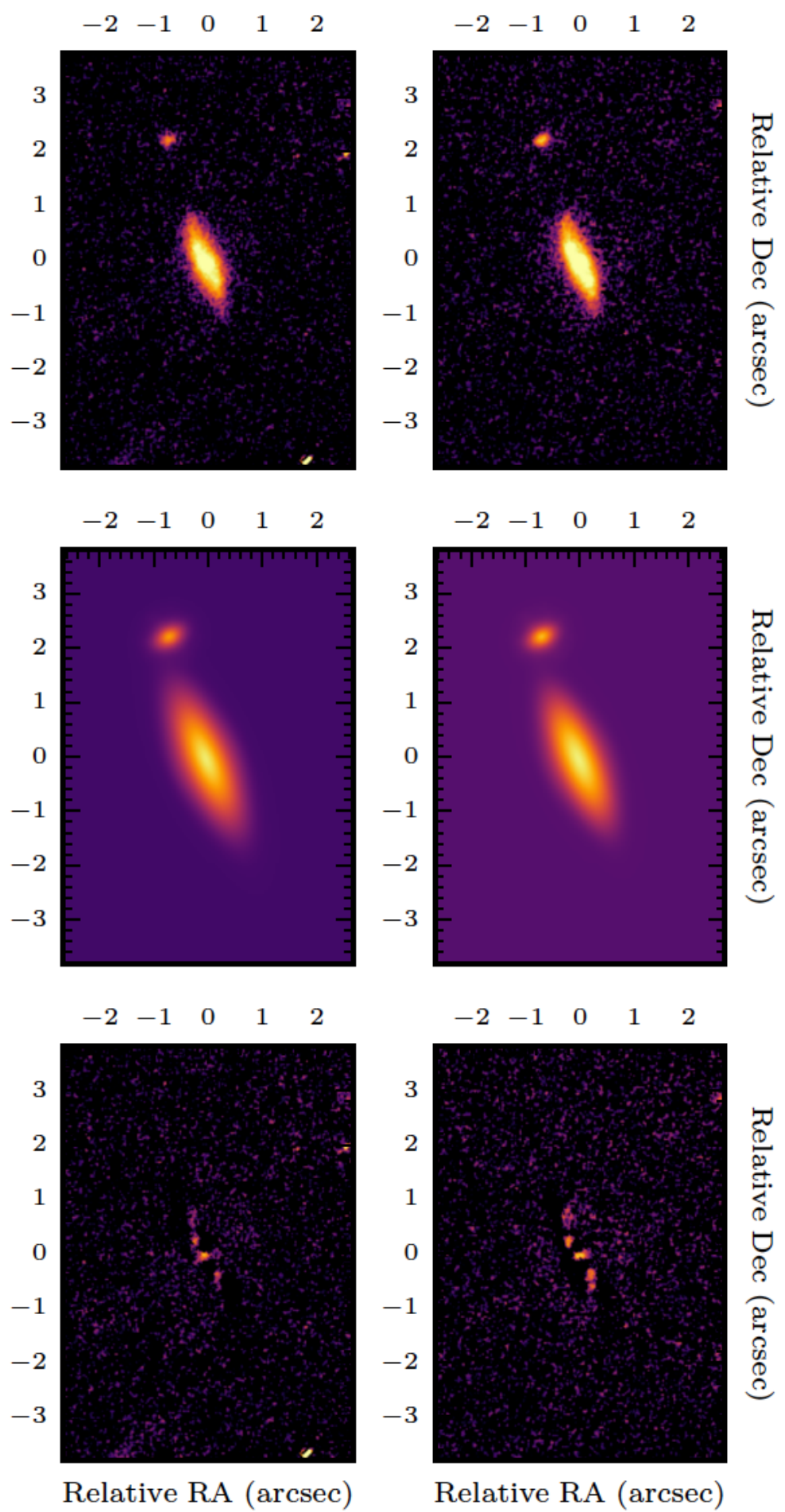

Fig. B.2. Morphology of the object \#8762. This object is a BLAGN at $z \sim 0.4$. It is a barred spiral galaxy (SB), whose bar appears visible in the residual images. First row: HST images of the object. Second row: GALFIT models. Third row: GALFIT residuals obtained by subtracting its model to the original image. First column: $V$ filter of HST (F814W) and second one: I filter (F606W). 

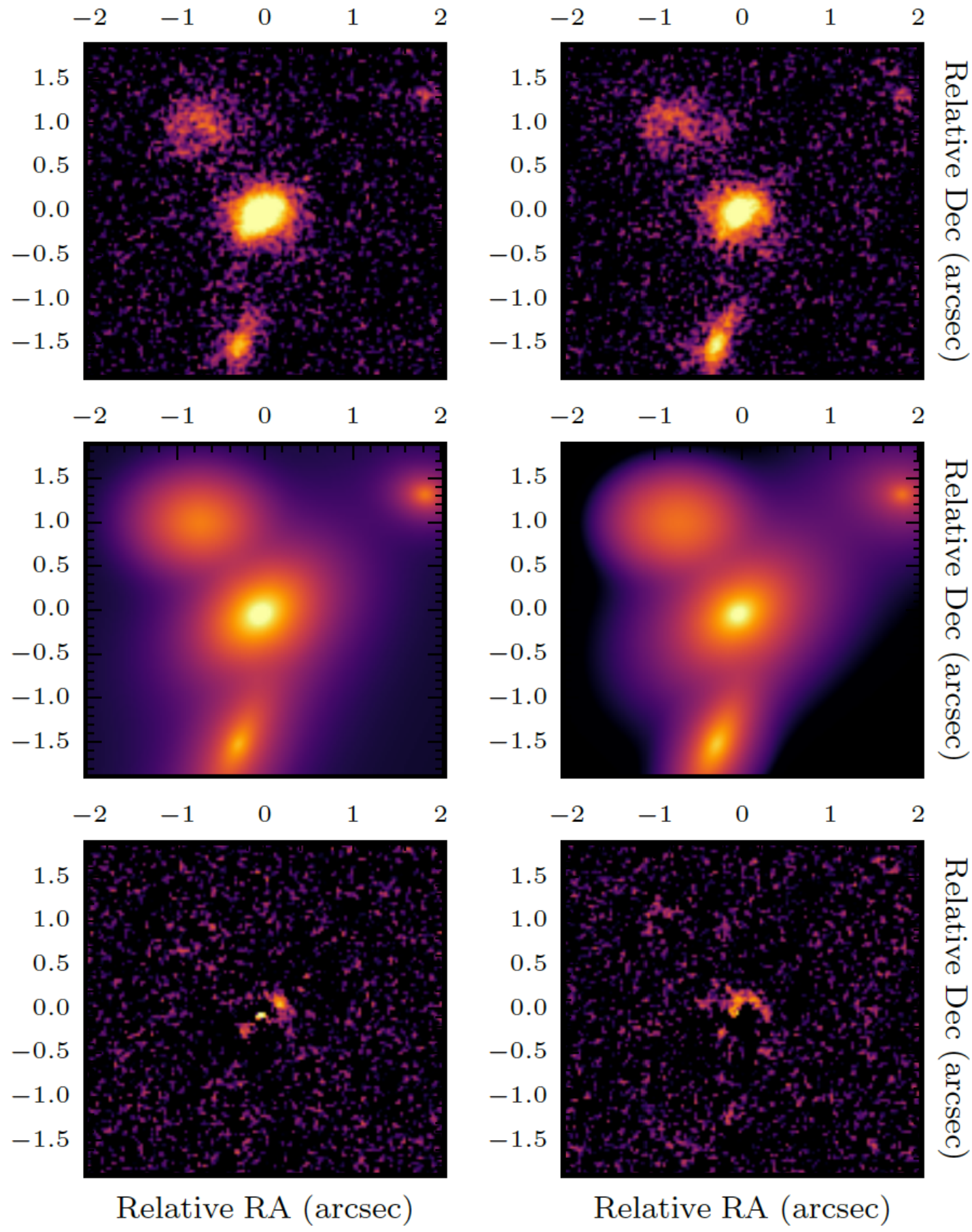

Fig. B.3. Morphology of the object \#2146. This object is a BLAGN at $z \sim 0.4$. It was classified as a late-type and flagged as a multiple object. First row: HST images of the object. Second row: GALFIT models. Third row: GALFIT residuals obtained by subtracting its model to the original image. First column: $V$ filter of HST (F814W) and second one: I filter (F606W). 

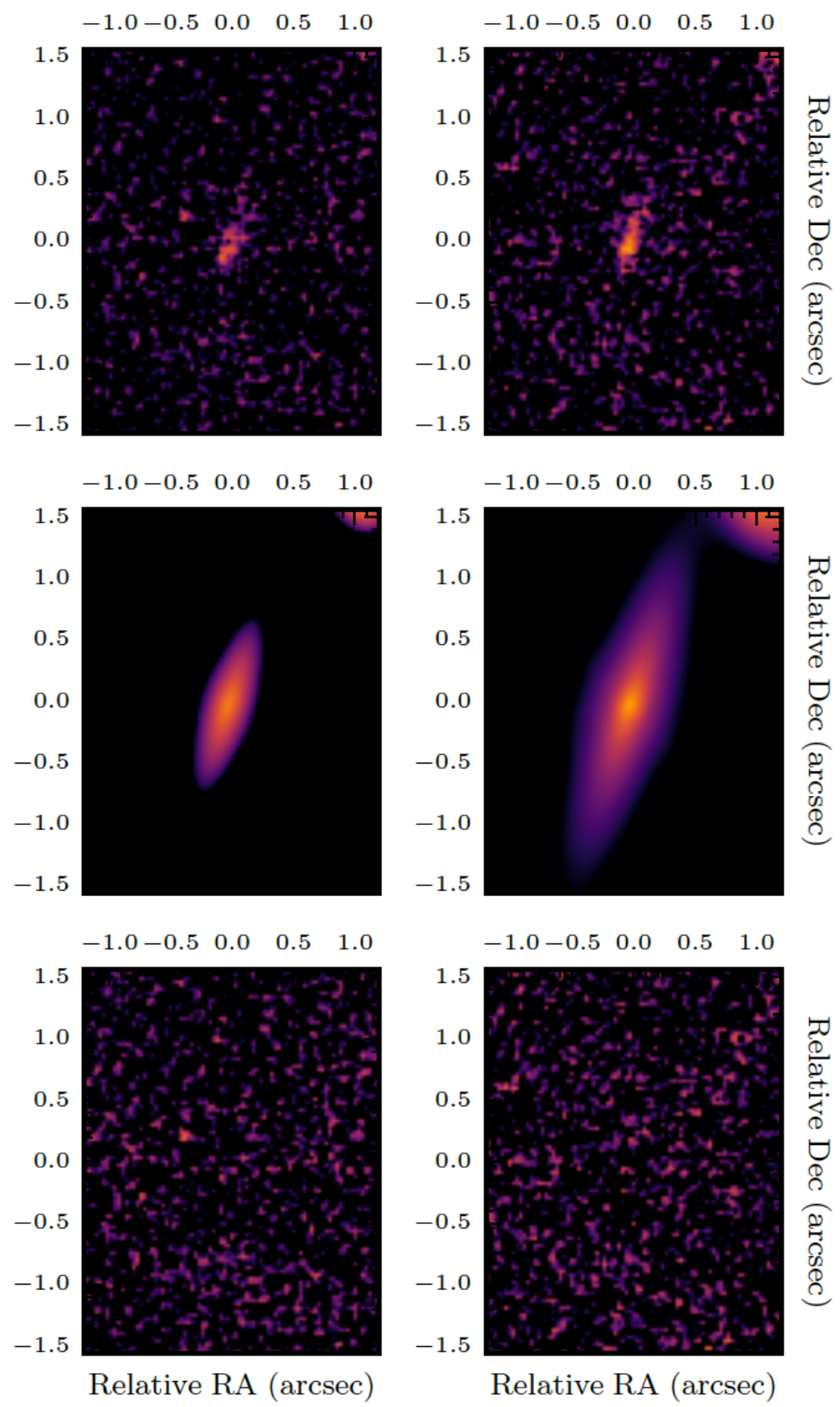

Fig. B.4. Morphology of the object \#3854. This object was classified as a NLAGN at $z \sim 0.4$ and was not assigned any morphological type (unclassifiable). First row: HST images of the object. Second row: GALFIT models. Third row: GALFIT residuals obtained by subtracting its model to the original image. First column: $V$ filter of HST (F814W) and second one: $I$ filter (F606W). 
M. Ramón-Pérez et al.: Active galactic nuclei in the OTELO survey
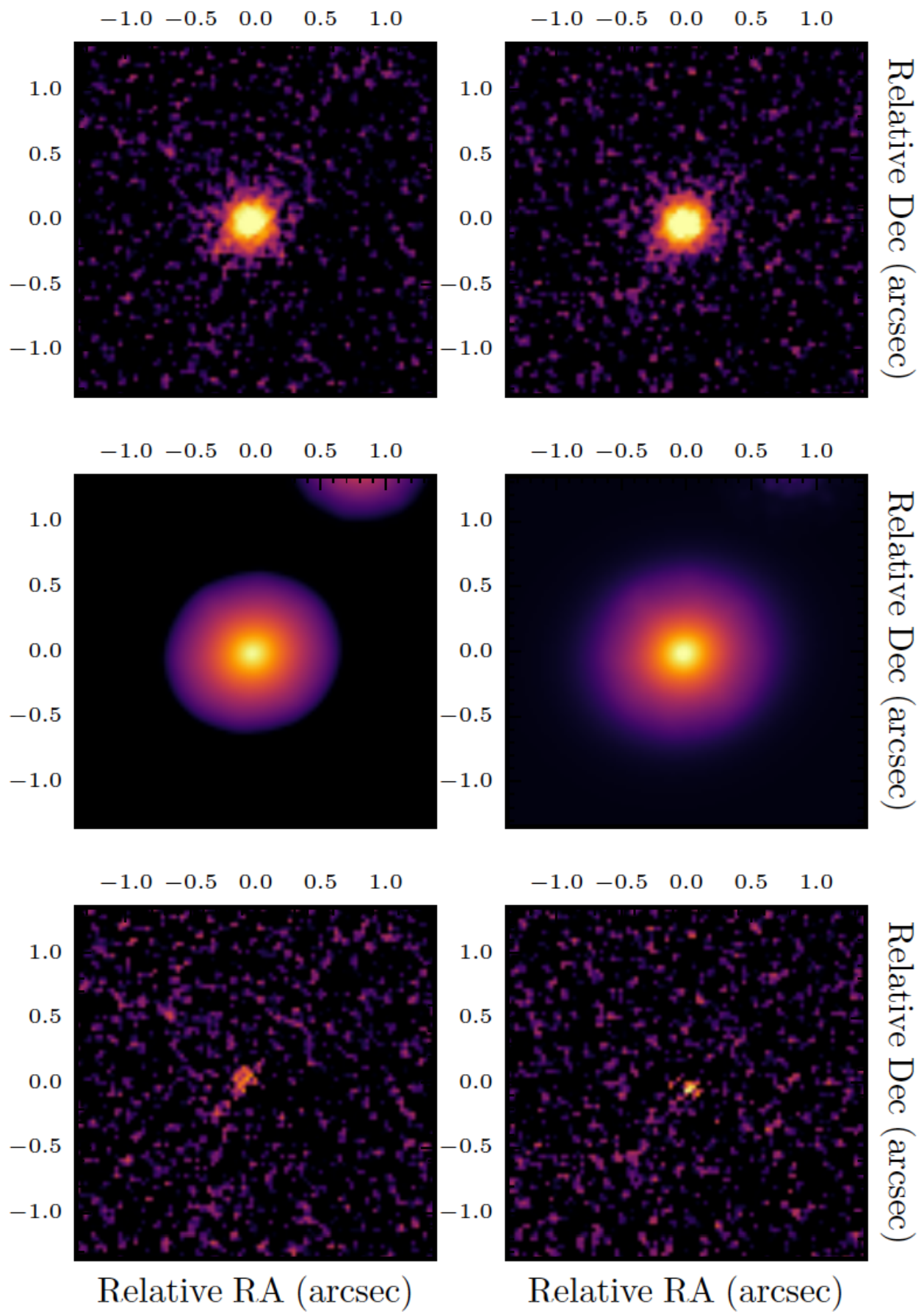

Fig. B.5. Morphology of the object \#6395. This object was classified as a NLAGN at $z \sim 0.4$ with a spheroidal morphology (early-type). First row: HST images of the object. Second row: GALFIT models. Third row: GALFIT residuals obtained by subtracting its model to the original image. First column: $V$ filter of HST (F814W) and second one: I filter (F606W). 

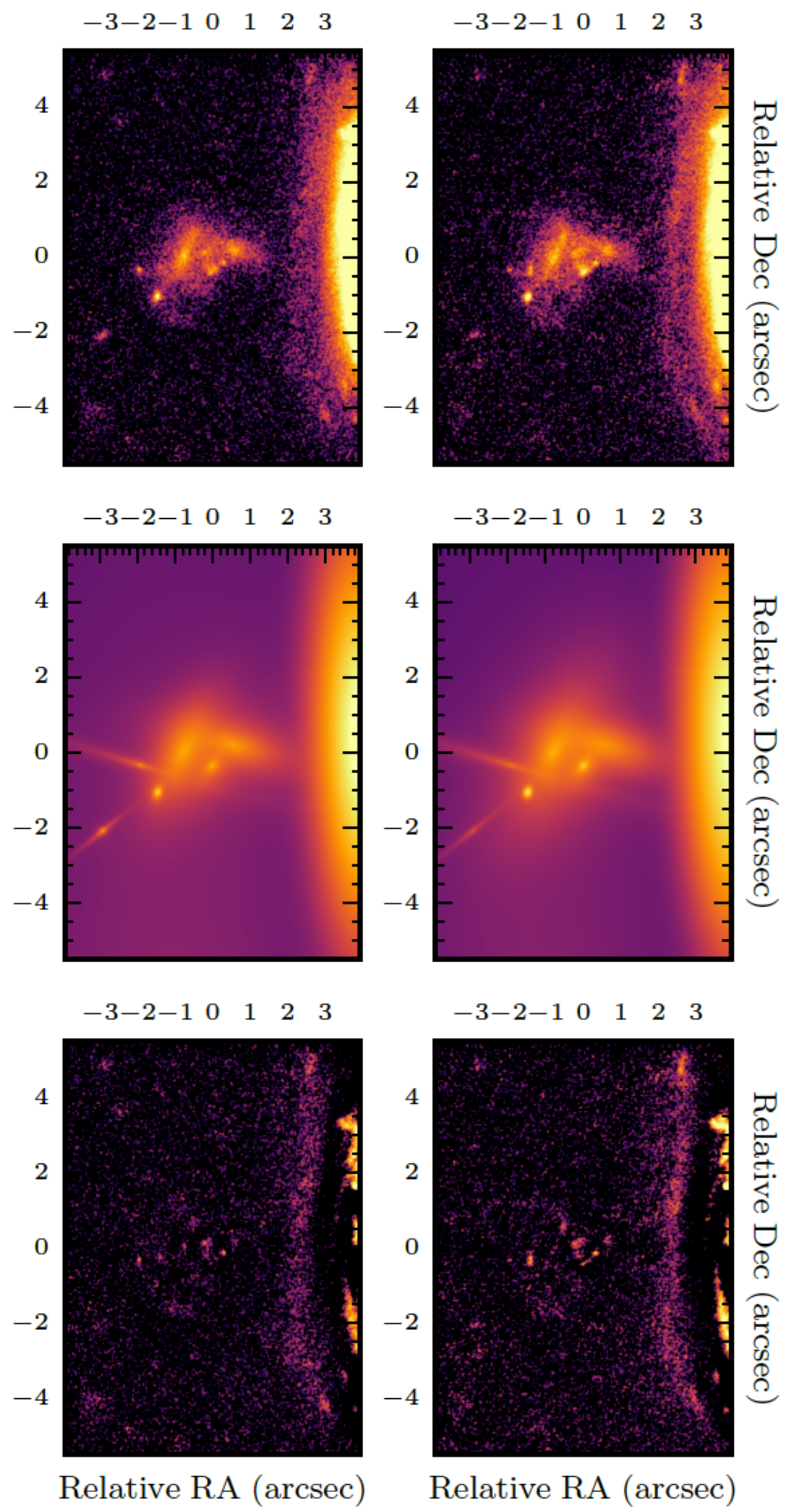

Fig. B.6. Morphology of the object \#5662. This object, selected as an AGN by its X-rays emission, is part of a system with multiple interacting components. Due to its complexity, it was not assigned any morphological type (unclassifiable). First row: HST images of the object. Second row: GALFIT models. Third row: GALFIT residuals obtained by subtracting its model to the original image. First column: $V$ filter of HST (F814W) and second one: $I$ filter (F606W). 
M. Ramón-Pérez et al.: Active galactic nuclei in the OTELO survey
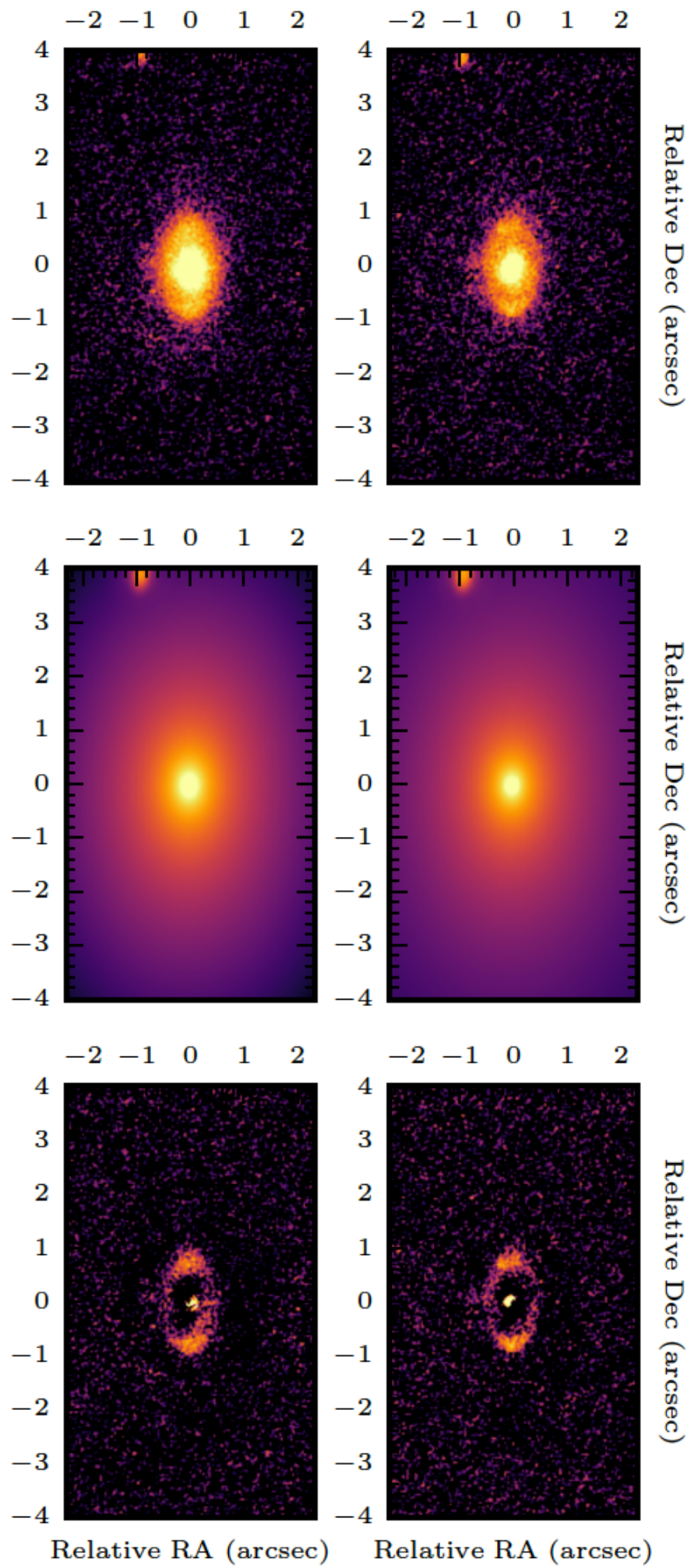

Fig. B.7. Morphology of the object \#3216. This object is an X-ray-selected AGN. It is a face-on spiral, whose arms are visible in the residual images (late-type). First row: HST images of the object. Second row: GALFIT models. Third row: GALFIT residuals obtained by subtracting its model to the original image. First column: $V$ filter of HST (F814W) and second one: I filter (F606W). 


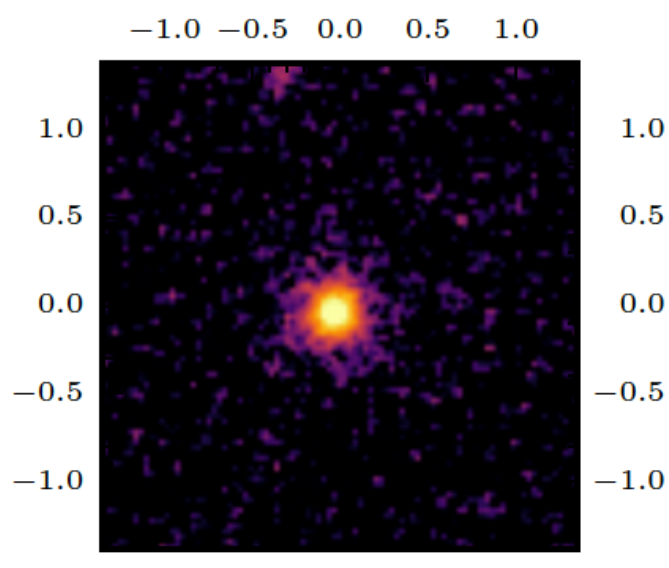

$\begin{array}{lllll}-1.0 & -0.5 & 0.0 & 0.5 & 1.0\end{array}$
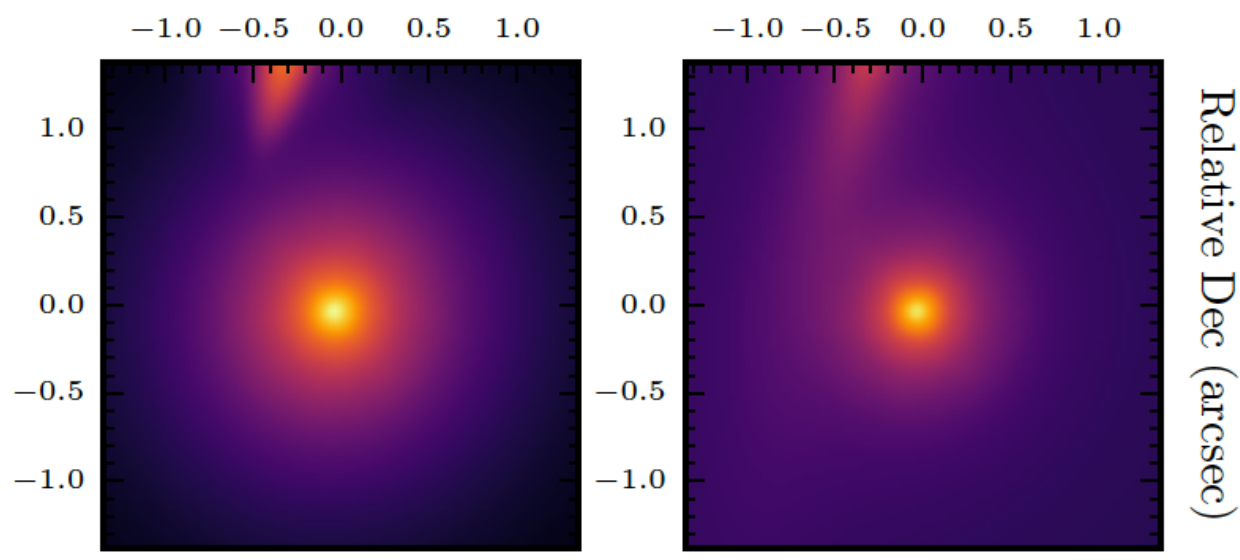

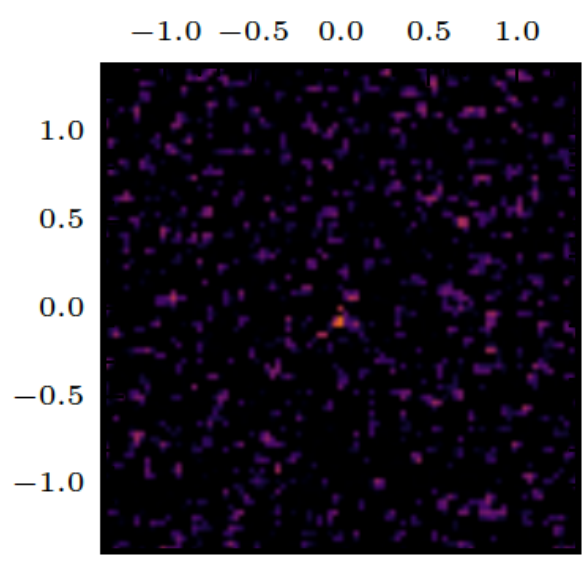

Relative RA (arcsec)

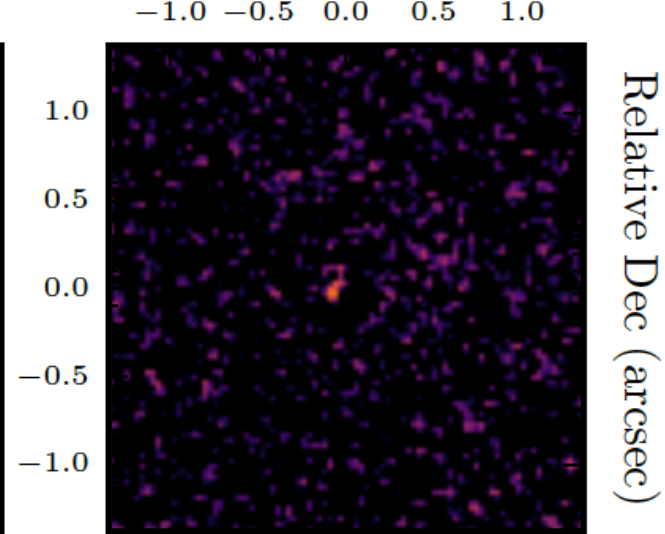

Relative RA (arcsec)

Fig. B.8. Morphology of the object \#6173. This object is an X-ray-selected AGN. It has a point-like appearance, and is probably a QSO, just like the object \#8351 shown in Fig. B.11. First row: HST images of the object. Second row: GALFIT models. Third row: GALFIT residuals obtained by subtracting its model to the original image. First column: $V$ filter of HST (F814W) and second one: I filter (F606W). 
M. Ramón-Pérez et al.: Active galactic nuclei in the OTELO survey
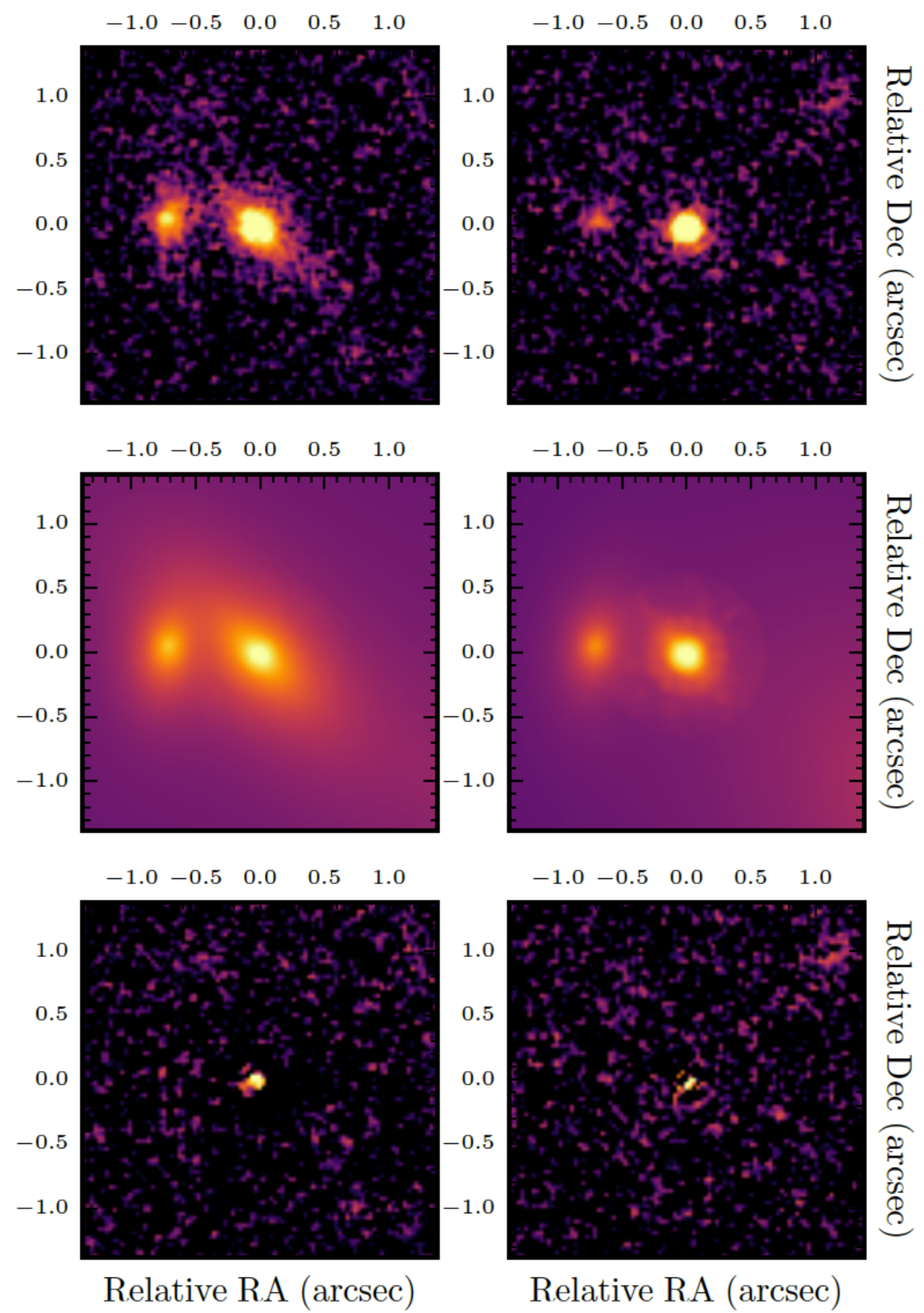

Fig. B.9. Morphology of the object \#5495. This object is an X-ray-selected AGN. It seems to be an early-type galaxy (probably lenticular, S0) with a companion. First row: HST images of the object. Second row: GALFIT models. Third row: GALFIT residuals obtained by subtracting its model to the original image. First column: $V$ filter of HST (F814W) and second one: $I$ filter (F606W). 

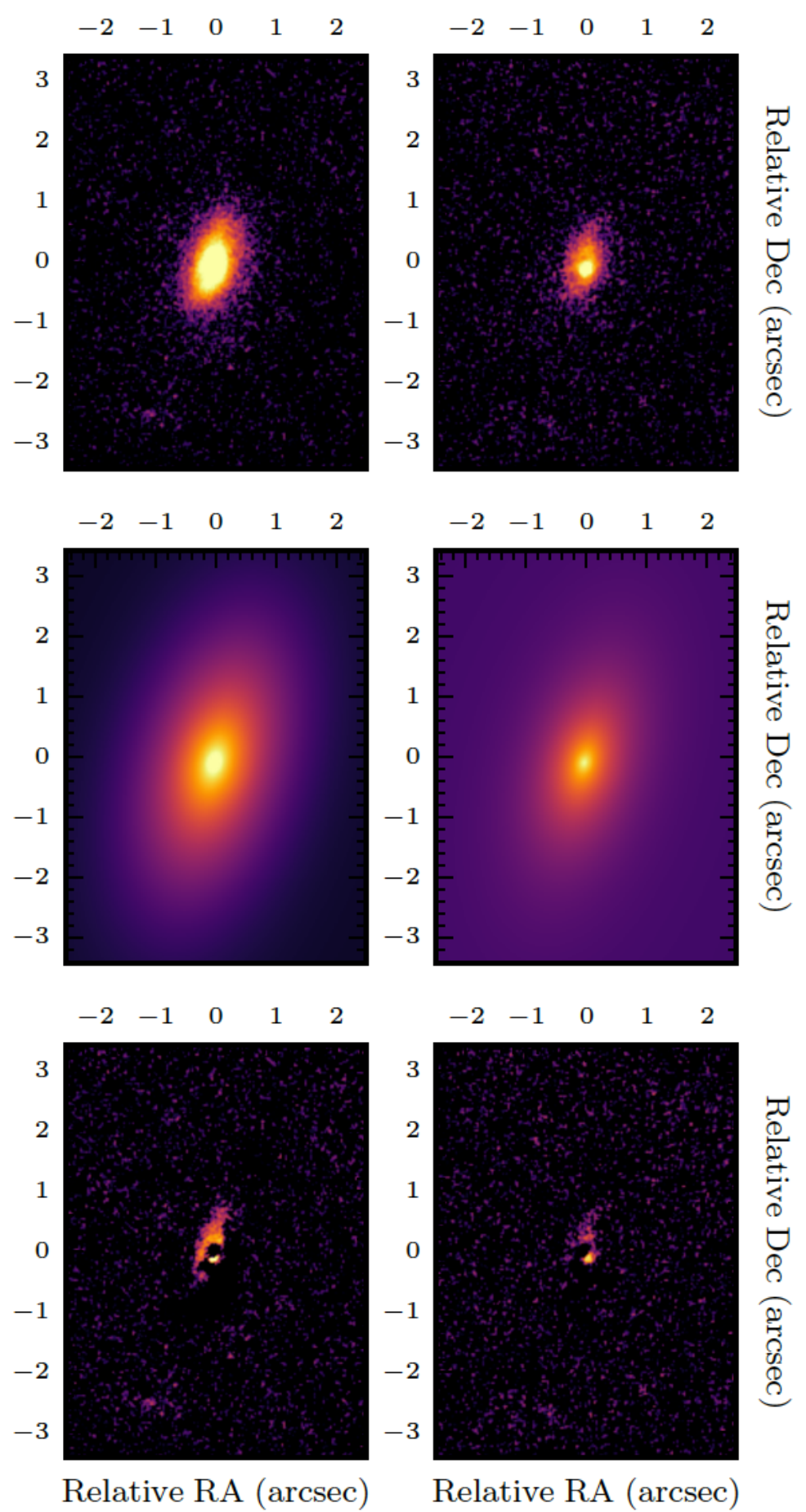

Fig. B.10. Morphology of the object \#8459. This object is an unobscured AGN (selected both in X-rays and IR) with a morphological disc type (late-type). First row: HST images of the object. Second row: GALFIT models. Third row: GALFIT residuals obtained by subtracting its model to the original image. First column: $V$ filter of HST (F814W) and second one: I filter (F606W). 

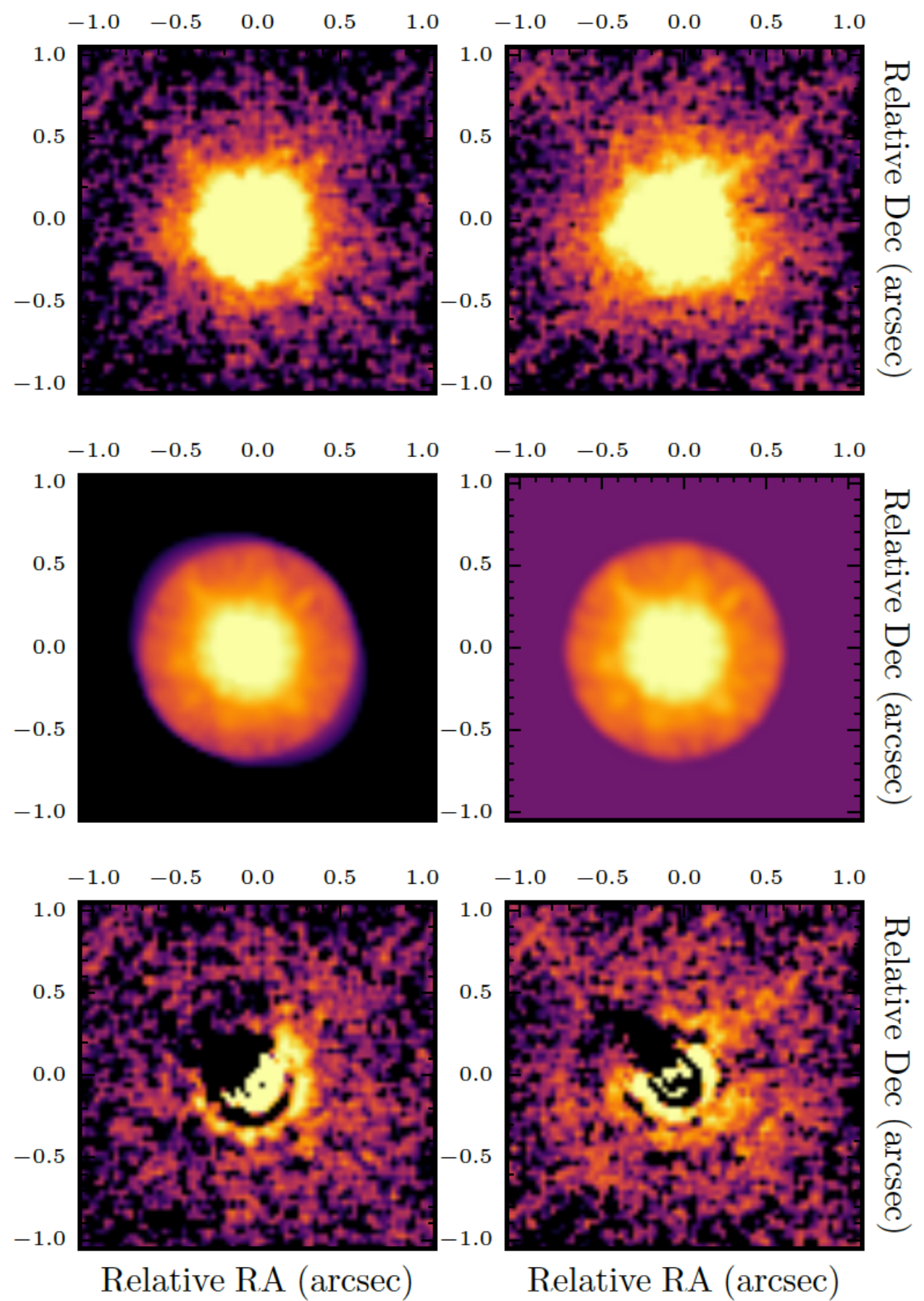

Fig. B.11. Morphology of the object \#8351. This object was selected as an AGN both in X-rays and MIR. It has a point-like appearance, and is probably a QSO, just like the object \#6173 shown in Fig. B.8. First row: HST images of the object. Second row: GALFIT models. Third row: GALFIT residuals obtained by subtracting its model to the original image. First column: $V$ filter of HST (F814W) and second one: I filter (F606W). 

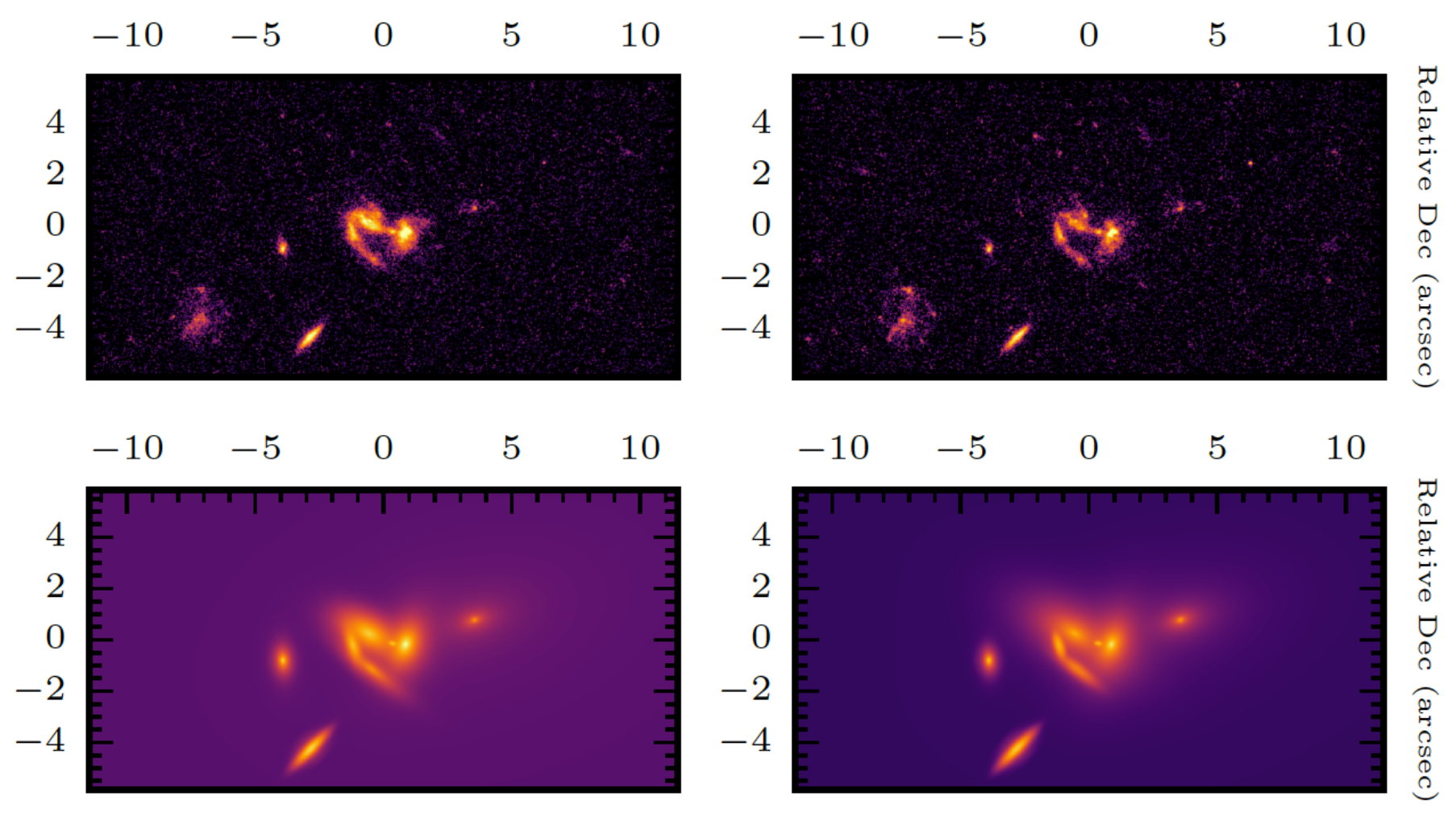

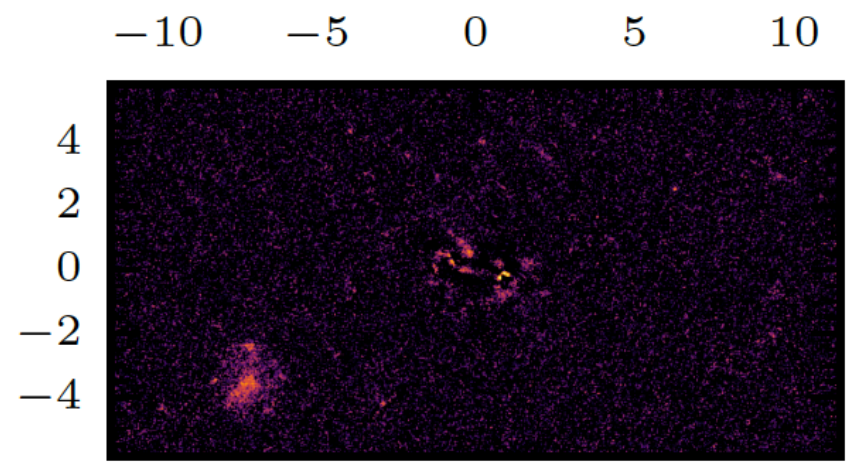

Relative RA (arcsec)

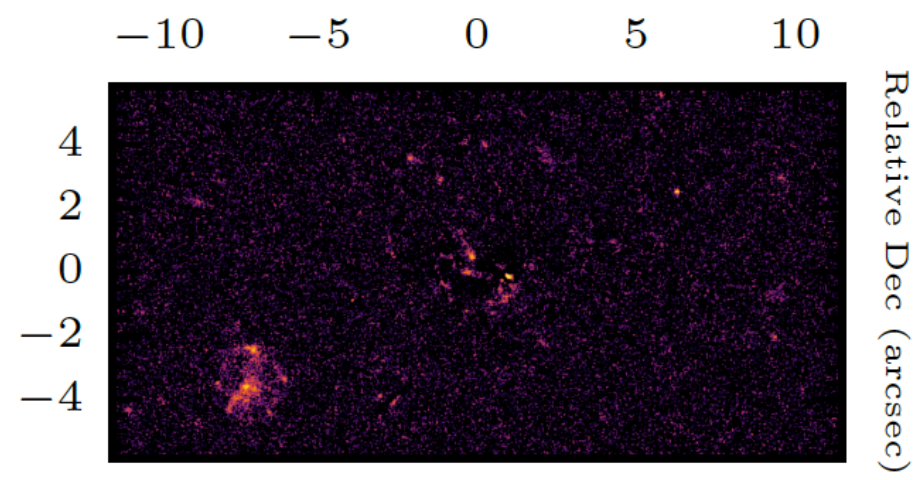

Relative RA (arcsec)

Fig. B.12. Morphology of the object \#11168. This system was selected by IR methods as an AGN. Two interacting spiral galaxies are visible. First row: HST images of the object. Second row: GALFIT models. Third row: GALFIT residuals obtained by subtracting its model to the original image. First column: $V$ filter of HST (F814W) and second one: $I$ filter (F606W). 

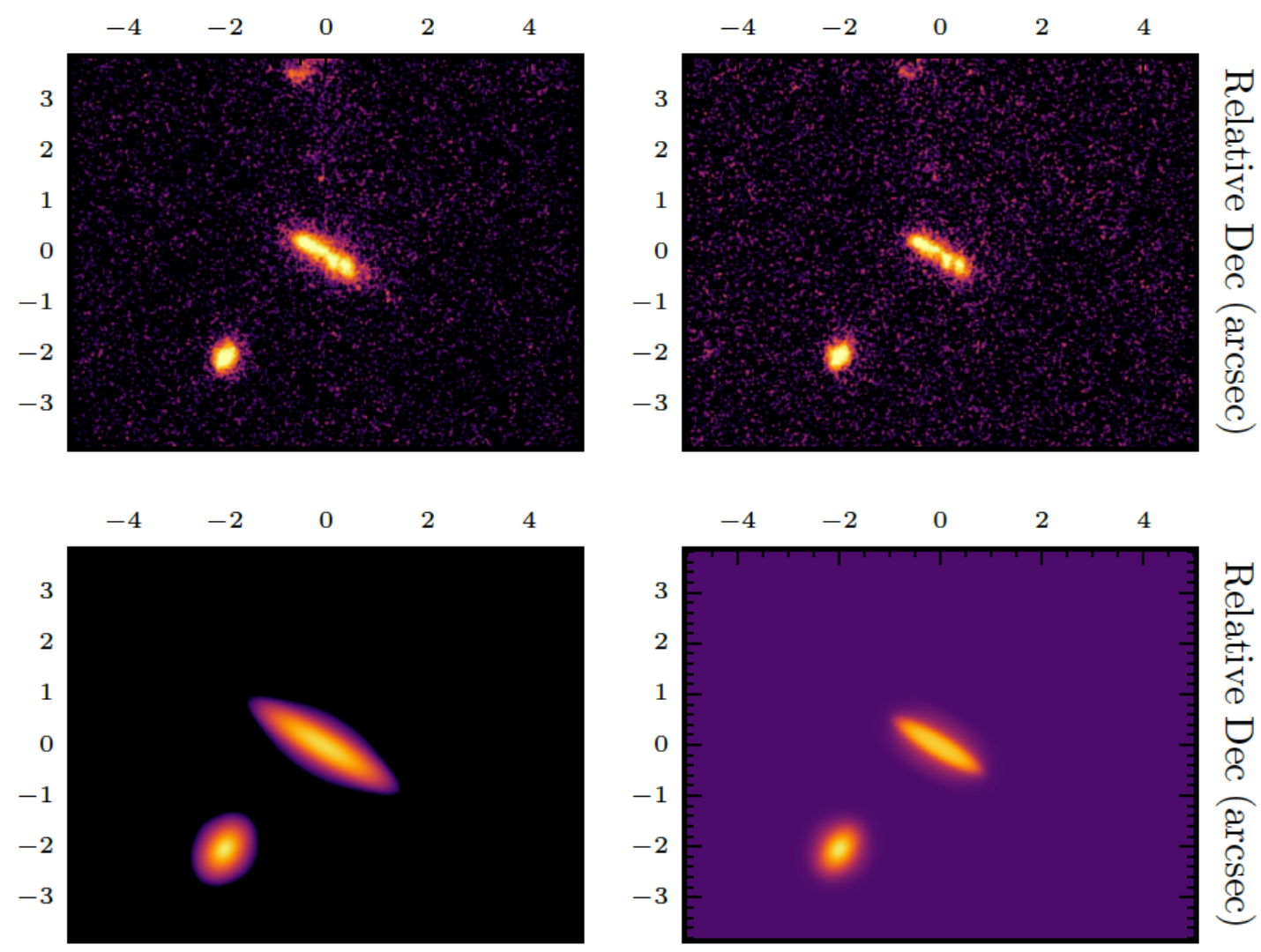

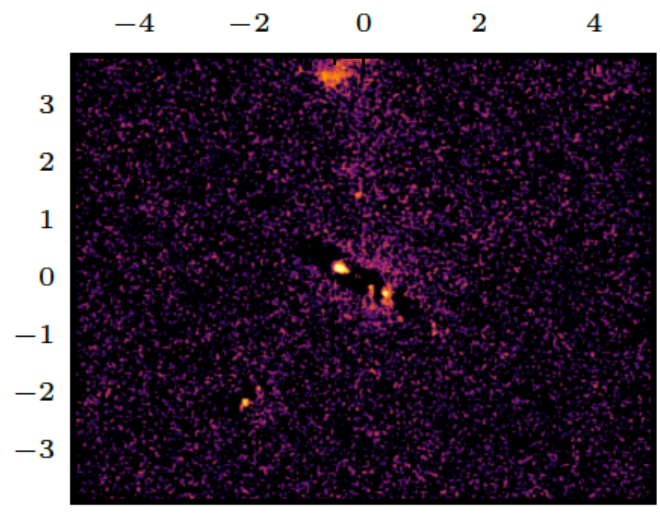

Relative RA (arcsec)

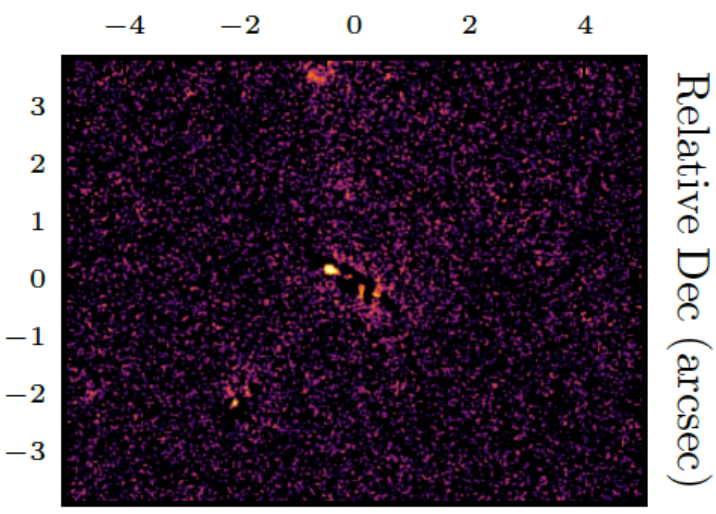

Relative RA (arcsec)

Fig. B.13. Morphology of the object \#7800. This object is an obscured AGN. It was selected with IR methods and classified as an irregular object (late-type), seemingly a chain-galaxy. First row: HST images of the object. Second row: GALFIT models. Third row: GALFIT residuals obtained by subtracting its model to the original image. First column: $V$ filter of HST (F814W) and second one: I filter (F606W). 

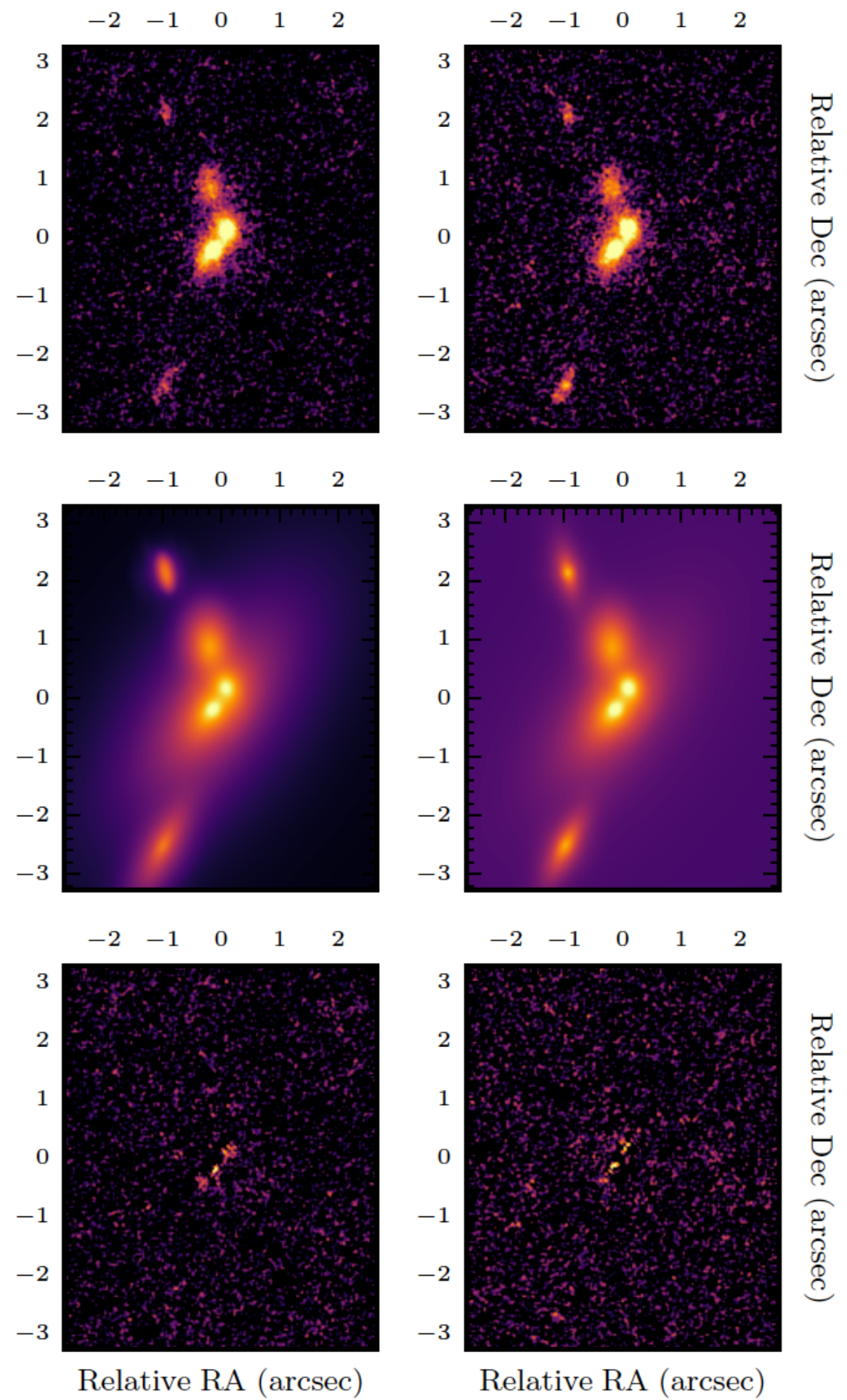

Fig. B.14. Morphology of the object \#10965. This object is an obscured AGN, selected only by IR methods, which seems to be interacting. It was classified as a late-type galaxy. First row: HST images of the object. Second row: GALFIT models. Third row: GALFIT residuals obtained by subtracting its model to the original image. First column: $V$ filter of HST (F814W) and second one: I filter (F606W). 
M. Ramón-Pérez et al.: Active galactic nuclei in the OTELO survey
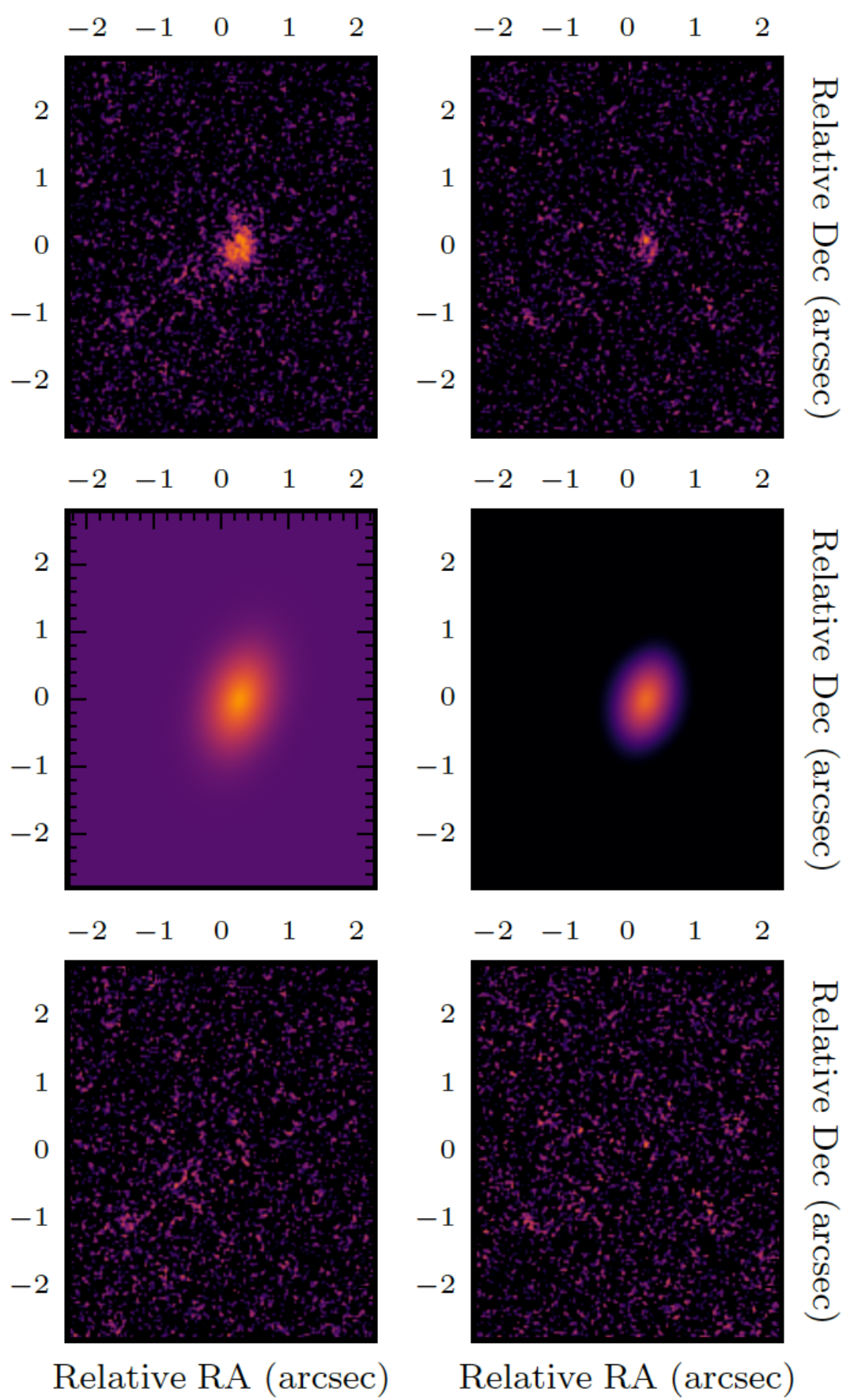

Fig. B.15. Morphology of the object \#7772. This object is a faint obscured AGN, classified as an irregular galaxy (late-type). First row: HST images of the object. Second row: GALFIT models. Third row: GALFIT residuals obtained by subtracting its model to the original image. First column: $V$ filter of HST (F814W) and second one: I filter (F606W). 